\title{
Curvature-Dimension Condition Meets Gromov's n-Volumic Scalar Curvature
}

Jialong DENG

Mathematisches Institut, Georg-August-Universität, Göttingen, Germany

E-mail: jialong.deng@mathematik.uni-goettingen.de

Received July 29, 2020, in final form January 23, 2021; Published online February 05, 2021

https://doi.org/10.3842/SIGMA.2021.013

\begin{abstract}
We study the properties of the $n$-volumic scalar curvature in this note. LottSturm-Villani's curvature-dimension condition $\mathrm{CD}(\kappa, n)$ was showed to imply Gromov's $n$-volumic scalar curvature $\geq n \kappa$ under an additional $n$-dimensional condition and we show the stability of $n$-volumic scalar curvature $\geq \kappa$ with respect to smGH-convergence. Then we propose a new weighted scalar curvature on the weighted Riemannian manifold and show its properties.
\end{abstract}

Key words: curvature-dimension condition; $n$-volumic scalar curvature; stability; weighted scalar curvature $\mathrm{Sc}_{\alpha, \beta}$

2020 Mathematics Subject Classification: 53C23

\section{Introduction}

The concept of lower bounded curvature on the metric space or the metric measure space has evolved to a rich theory due to Alexandrov's insight. The stability of Riemannian manifolds with curvature bounded below is another deriving force to extend the definition of the curvature bounded below to a broader space. However, the scalar curvature (of Riemannian metrics) bounded below was yet absent from this picture. Gromov proposed a synthetic treatment of scalar curvature bounded below, which was called the $n$-volumic scalar curvature bounded below, and offered some pertinent conjectures in [18, Section 26]. Motivated by the $\operatorname{CD}(\kappa, n)$ condition, we add an $n$-dimension condition to the Gromov's definition and introduce the definition of $\mathrm{Sc}_{\alpha, \beta}$ on the smooth metric measure space. Details will be given later.

Theorem 1.1. Assume that the metric measure space $\left(X^{n}, d, \mu\right)$ satisfies $n$-dimensional condition and the curvature-dimension condition $\mathrm{CD}(\kappa, n)$ for $\kappa \geq 0$ and $n \geq 2$, then $\left(X^{n}, d, \mu\right)$ satisfies $\operatorname{Sc}^{\operatorname{vol}_{n}}\left(X^{n}\right) \geq n \kappa$.

Theorem 1.2. If compact metric measure spaces $\left(X_{i}^{n}, d_{i}, \mu_{i}\right)$ with $\operatorname{Sc}^{\mathrm{vol}_{n}}\left(X_{i}^{n}\right) \geq \kappa \geq 0$ and $S C$-radius $r_{x_{i}^{n}} \geq R>0$ and $\left(X_{i}^{n}, d_{i}, \mu_{i}\right)$ strongly measured Gromov-Hausdorff converge to the compact metric measure space $\left(X^{n}, d, \mu\right)$ with $n$-dimensional condition, then $X^{n}$ also satisfies $\mathrm{Sc}^{\operatorname{vol}_{n}}\left(X^{n}\right) \geq \kappa$ and the $S C$-radius $r_{X^{n}} \geq R$.

Theorem 1.3. Let $\left(M^{n}, g, \mathrm{e}^{-f} \mathrm{dVol}_{g}\right)$ be the closed smooth metric measure space with $\mathrm{Sc}_{\alpha, \beta}>0$, then we have the following conclusions:

1. If $M^{n}$ is a spin manifold, $\alpha \in \mathbb{R}$ and $\beta \geq \frac{|\alpha|^{2}}{4}$, then the harmonic spinors of $M^{n}$ vanish.

2. If the dimension $n \geq 3, \alpha \in \mathbb{R}$ and $\beta \geq \frac{(n-2)|\alpha|^{2}}{4(n-1)}$, then there is a metric $\tilde{g}$ conformal to $g$ with positive scalar curvature.

This paper is a contribution to the Special Issue on Scalar and Ricci Curvature in honor of Misha Gromov on his 75th Birthday. The full collection is available at https://www.emis.de/journals/SIGMA/Gromov.html 
3. If the dimension $n \geq 3, \alpha=2, \beta \geq \frac{n-2}{n-1}$ and $\left(N^{n-1}, \bar{g}\right)$ is the compact $L_{f}$-stable minimal hypersurface of $\left(M^{n}, g, \mathrm{e}^{-f} \mathrm{dVol}_{g}\right)$, then there exists a PSC-metric conformal to $\bar{g}$ on $N^{n-1}$, where $\bar{g}$ is the induced metric of $g$ on $N^{n-1}$.

4. Assume $M^{n}$ is a spin manifold and there exists a smooth 1-contracting map $h:\left(M^{n}, g\right) \rightarrow$ $\left(S^{n}, g_{\mathrm{st}}\right)$ of non-zero degree. If $\alpha \in \mathbb{R}, \beta \geq \frac{|\alpha|^{2}}{4}$ and $\mathrm{Sc}_{\alpha, \beta} \geq n(n-1)$, then $h$ is an isometry between the metrics $g$ and $g_{\mathrm{st}}$.

The paper is organized as follows. In Section 2, we introduce the notions and show that $\mathrm{CD}(\kappa, n)$ implies $\mathrm{Sc}^{\mathrm{vol}_{n}} \geq(n-1) \kappa$. In Section 3, we show the stability of spaces with $\mathrm{Sc}^{\mathrm{vol}_{n}} \geq \kappa$. In Section 4 , we present the properties of the smooth metric measure space with $\mathrm{Sc}_{\alpha, \beta}>0$.

\section{CD meets $n$-volumic scalar curvature}

The $n$-dimensional Aleksandrov space with curvature $\geq \kappa$ equipped with the volume-measure satisfies Lott-Villani-Sturm's weak curvature-dimension condition for dimension $n$ and curvature $(n-1) \kappa$, i.e., $\mathrm{CD}((n-1) \kappa, n)$, was shown by Petrunin for $\kappa=0$ (and said that for general curvature $\geq \kappa$ the result followed in a similar way) [32] and then Zhang-Zhu investigated the general case [43]. We will modify Gromov's definition of $n$-volumic scalar curvature bounded below in [18, Section 26] to fill the picture, which means Lott-Sturm-Villani's Ricci curvature $\geq 0$ implies Gromov's scalar curvature $\geq 0$.

The metric measure space (mm-space) $X=(X, d, \mu)$ means that $d$ is the complete separable length metric on $X$ and $\mu$ is the locally finite full support Borel measure on $X$ equipped with its Borel $\sigma$-algebra. Say that an mm-space $X=(X, d, \mu)$ is locally volume-wise smaller (or not greater) than another such space $X^{\prime}=\left(X^{\prime}, d^{\prime}, \mu^{\prime}\right)$ and write $X<_{\mathrm{vol}} X^{\prime}\left(X \leq_{\mathrm{vol}} X^{\prime}\right)$, if all $\epsilon$-balls in $X$ are smaller (or not greater) than the $\epsilon$-balls in $X^{\prime}, \mu\left(B_{\epsilon}(x)\right)<\mu^{\prime}\left(B_{\epsilon}\left(x^{\prime}\right)\right)\left(\mu\left(B_{\epsilon}(x)\right) \leq\right.$ $\mu^{\prime}\left(B_{\epsilon}\left(x^{\prime}\right)\right)$, for all $x \in X, x^{\prime} \in X^{\prime}$ and the uniformly small $\epsilon$ which depends on $X$ and $X^{\prime}$.

From now on, the Riemannian 2-sphere $\left(S^{2}(\gamma), d_{S}\right.$, $\left.\operatorname{vol}_{S}\right)$ is endowed with round metric such that the scalar curvature equal to $2 \gamma^{-2},\left(\mathbf{R}^{n-2}, d_{E}, \operatorname{vol}_{E}\right)$ is endowed with Euclidean metric with flat scalar curvature and the product manifold $S^{2}(\gamma) \times \mathbf{R}^{n-2}$ is endowed with the Pythagorean product metrics $d_{S \times E}:=\sqrt{d_{S}^{2}+d_{E}^{2}}$ and the volume $\operatorname{vol}_{S \times E}:=\operatorname{vol}_{S} \otimes \operatorname{vol}_{E}$.

Thus, we have $S^{2}(\gamma)<_{\text {vol }} \mathbf{R}^{2}$. If $0<\gamma_{1}<\gamma_{2}$, then $S^{2}\left(\gamma_{1}\right)<_{\text {vol }} S^{2}\left(\gamma_{2}\right)$. Furthermore, $S^{2}(\gamma) \times \mathbf{R}^{n-2}<_{\mathrm{vol}} \mathbf{R}^{n}$. If $0<\gamma_{1}<\gamma_{2}$, then $S^{2}\left(\gamma_{1}\right) \times \mathbf{R}^{n-2}<_{\mathrm{vol}} S^{2}\left(\gamma_{2}\right) \times \mathbf{R}^{n-2}$.

Definition 2.1 (Gromov's $n$-volumic scalar curvature). Gromov's $n$-volumic scalar curvature of $X$ is bounded below by 0 for $X=(X, d, \mu)$ if $X$ is locally volume-wise not greater than $\mathbf{R}^{n}$.

Gromov's $n$-volumic scalar curvature of $X$ bounds from below by $\kappa>0$ for $X=(X, d, \mu)$ if $X$ is locally volume-wise smaller than $S^{2}(\gamma) \times \mathbf{R}^{n-2}$ for all $\gamma>\sqrt{\frac{2}{\kappa}}$, i.e., $X<_{\text {vol }} S^{2}(\gamma) \times \mathbf{R}^{n-2}$ and $\gamma>\sqrt{\frac{2}{\kappa}}$, where $S^{2}(\gamma) \times \mathbf{R}^{n-2}=\left(S^{2}(\gamma) \times \mathbf{R}^{n-2}, d_{S \times E}, \operatorname{vol}_{S \times E}\right)$.

The $n$-volumic scalar curvature is sensitive to the scaling of the measure, but the curvature condition $\mathrm{CD}(\kappa, n)$ of Lott-Villani-Sturm [38, Definition 1.3] is invariant up to scalars of the measure only [38, Proposition 1.4(ii)]. Therefore, the $n$-dimensional condition needs to be put into the definition of Gromov's $n$-volumic scalar curvature. In fact, the $n$-dimensional condition is the special case of Young's point-wise dimension in dynamical systems [41, Theorem 4.4].

Definition 2.2 ( $n$-dimensional condition). For given positive natural number $n$, the mm-space $X=(X, d, \mu)$ satisfies the $n$-dimensional condition if

$$
\lim _{r \rightarrow 0} \frac{\mu\left(B_{r}(x)\right)}{\operatorname{vol}_{E}\left(B_{r}\left(\mathbf{R}^{n}\right)\right)}=1
$$


for every $x \in X$, where $B_{r}\left(\mathbf{R}^{n}\right)$ is the closed $r$-ball in the Euclidean space $\mathbf{R}^{n}$ and the $B_{r}(x)$ is the closed $r$-ball with the center $x \in X$.

From now on, the superscript of $n$ in the space $X^{n}$ means the mm-space $\left(X^{n}, d, \mu\right)$ satisfies $n$-dimensional condition.

Note that a closed smooth $n$-manifold $M^{n}(n \geq 3)$ admits a Riemannian metric with constant negative scalar curvature and a Riemannian metric of non-negative scalar curvature which is not identically zero, then by a conformal change of the metric we get a metric of positive scalar curvature according to Kazdan-Warner theorem [25]. Furthermore, if there is a scalarflat Riemannian metric $g$ on $M^{n}$, but $g$ is not Ricci-flat metric, then $g$ can be deformed to a metric with positive scalar curvature according to Kazdan theorem [24, Theorem B] or by using Ricci-flow with an easy argument. Hence we will focus more on promoting the positive scalar curvature to positive $n$-volumic scalar curvature.

Definition 2.3 ( $n$-volumic scalar curvature). Assume $X^{n}=\left(X^{n}, d, \mu\right)$ is the compact mm-space and satisfies the $n$-dimensional condition, we call

1. the $n$-volumic scalar curvature of $X^{n}$ is positive, i.e., $\operatorname{Sc}^{\operatorname{vol}_{n}}\left(X^{n}\right)>0$, if there exists $r_{X^{n}}>0$ such that the measures of $\epsilon$-balls in $X^{n}$ are smaller than the volumes of $\epsilon$-balls in $\mathbf{R}^{n}$ for $0<\epsilon \leq r_{X^{n}}$.

2. the $n$-volumic scalar curvature of $X^{n}$ is bounded below by 0 , i.e., $\operatorname{Sc}^{\operatorname{vol}_{n}}\left(X^{n}\right) \geq 0$, if there exists $r_{X^{n}}>0$ such that the measures of $\epsilon$-balls in $X^{n}$ are not greater than the volumes of $\epsilon$-balls in $\mathbf{R}^{n}$ for $0<\epsilon \leq r_{X^{n}}$.

The $r_{X^{n}}$ is called scalar curvature radius (SC-radius) of $X^{n}$ for $\operatorname{Sc}^{\operatorname{vol}_{n}}\left(X^{n}\right) \geq 0$.

3. the $n$-volumic scalar curvature of $X^{n}$ is bounded below by $\kappa>0$, i.e., $\operatorname{Sc}^{\operatorname{vol}_{n}}\left(X^{n}\right) \geq \kappa>0$, if, for any $\gamma$ with $\gamma>\sqrt{\frac{2}{\kappa}}$, there exists $r_{X^{n}, \gamma}>0$ such that the measures of $\epsilon$-balls in $X^{n}$ are smaller than the volumes of $\epsilon$-balls in $S^{2}(\gamma) \times \mathbf{R}^{n-2}$ for $0<\epsilon \leq r_{X^{n}, \gamma}$.

We call $r_{X^{n}}:=\inf _{\gamma>\sqrt{\frac{2}{\kappa}}} r_{X^{n}, \gamma}$ is the SC-radius of $X^{n}$ for $\operatorname{Sc}^{\operatorname{vol}_{n}}\left(X^{n}\right) \geq \kappa>0$.

In particular, we will focus on the case of inf $r_{X^{n}, \gamma} \neq 0$ for stability in Section 3. $\gamma>\sqrt{\frac{2}{\kappa}}$

If the mm-space $X^{n}$ is locally compact, then the definition of the $n$-volumic scalar curvature bounded below only modifies the definition of the $r_{X^{n}, \gamma}>0$ to a positive continuous function of $X^{n}$.

Two mm-spaces $\left(X^{n}, d, \mu\right)$ and $\left(X_{1}^{n}, d_{1}, \mu_{1}\right)$ are isometric if there exists a one-to-one map $f: X^{n} \rightarrow X_{1}^{n}$ such that $d_{1}(f(a), f(b))=d(a, b)$ for $a$ and $b$ are in $X^{n}$ and $f_{*} \mu=\mu_{1}$, where $f_{*} \mu$ is the push-forward measure, i.e., $f_{*} \mu(U)=\mu\left(f^{-1}(U)\right)$ for a measureable subset $U \subset X_{1}^{n}$. If $X^{n}$ satisfies $\operatorname{Sc}^{\operatorname{vol}_{n}}\left(X^{n}\right) \geq \kappa \geq 0$, then each mm-space $\left(X_{1}^{n}, d_{1}, \mu_{1}\right)$ that is isometric to $\left(X^{n}, d, \mu\right)$ also satisfies $\operatorname{Sc}^{\operatorname{vol}_{n}}\left(X_{1}^{n}\right) \geq \kappa \geq 0$.

Proposition 2.4. Let $g$ be a $C^{2}$-smooth Riemannian metric on a closed oriented $n$-manifold $M^{n}$ with induced metric measure space $\left(M^{n}, d_{g}, \mathrm{dVol}_{g}\right)$, then the scalar curvature of $g$ is positive, $\mathrm{Sc}_{g}>0$, if and only if $\mathrm{Sc}^{\mathrm{vol}_{n}}\left(M^{n}\right)>0$, and $\mathrm{Sc}_{g} \geq \kappa>0$ if and only if $\mathrm{Sc}^{\mathrm{vol}_{n}}\left(M^{n}\right) \geq \kappa>0$.

Proof. For a $C^{2}$-smooth Riemannian metric $g$, one has

$$
\mathrm{dVol}_{g}\left(B_{r}(x)\right)=\operatorname{vol}_{E}\left(B_{r}\left(\mathbf{R}^{n}\right)\right)\left[1-\frac{\mathrm{Sc}_{g}(x)}{6(n+2)} r^{2}+O\left(r^{4}\right)\right]
$$

for $B_{r}(x) \subset M^{n}$ as $r \rightarrow 0$. Hence $\left(M^{n}, d_{g}, \mathrm{dVol}_{g}\right)$ satisfies the $n$-dimensional condition. 
If we have $\mathrm{Sc}_{g}>0$, then, since $M^{n}$ is compact, there exists $r_{M^{n}}>0$, so that $\mathrm{dVol}_{g}\left(B_{r}(x)\right)<$ $\operatorname{vol}_{E}\left(B_{r}\left(\mathbf{R}^{n}\right)\right)$ for all $0<r \leq r_{M^{n}}$. On the other hand, if there exists $r_{M^{n}}>0$ such that $\mathrm{dVol}_{g}\left(B_{r}(x)\right)<\operatorname{vol}_{E}\left(B_{r}\left(\mathbf{R}^{n}\right)\right)$ for all $0<r \leq r_{M^{n}}$, then $\mathrm{Sc}_{g}$ must be greater than 0 .

If $\mathrm{Sc}^{\operatorname{vol}_{n}}\left(M^{n}\right) \geq \kappa>0$, then $\mathrm{Sc}_{g} \geq \kappa>0$. Otherwise, assume there exist small $\epsilon>0$ such that $\mathrm{Sc}_{g} \geq \kappa-\epsilon>0$. That means that there exists a point $x_{0}$ in $M^{n}$ such that $\operatorname{Sc}_{g}\left(x_{0}\right)=\kappa-\epsilon$, as $M^{n}$ is compact and the scalar curvature is a continuous function on $M^{n}$. Thus, we can find a small $r$-ball $B_{r}\left(x_{0}\right)$ such that the volume of $B_{r}\left(x_{0}\right)$ is greater than the volume of the $r$-ball in the $S^{2}(\gamma) \times \mathbf{R}^{n-2}$ for $\gamma=\sqrt{\frac{2}{\kappa-\frac{\epsilon}{2}}}$, which is a contradiction.

On the other hand, $\mathrm{Sc}_{g} \geq \kappa>0$ implies $\mathrm{Sc}^{\operatorname{vol}_{n}}\left(M^{n}\right) \geq \kappa>0$. Assume $\operatorname{Sc}_{g}\left(x_{1}\right)=\kappa$ for some $x_{1} \in M^{n}$, then there exists $r_{1}$ such that $\mathrm{dVol}_{g}\left(B_{r_{1}}(x)\right) \leq \mathrm{dVol}_{g}\left(B_{r_{1}}\left(x_{1}\right)\right)$ for $r_{1}$-balls in $M^{n}$ and

$$
\mathrm{dVol}_{g}\left(B_{r}\left(x_{1}\right)\right)=\operatorname{vol}_{E}\left(B_{r}\left(\mathbf{R}^{n}\right)\right)\left[1-\frac{\kappa}{6(n+2)} r^{2}+O\left(r^{4}\right)\right]
$$

as $r \rightarrow 0$. Thus, for any $\gamma$ with $\gamma>\sqrt{\frac{2}{\kappa}}$, there exists $r_{M^{n}, \gamma}>0$ such that the measures of $\epsilon$-balls in $M^{n}$ are smaller than the volumes of $\epsilon$-balls in $S^{2}(\gamma) \times \mathbf{R}^{n-2}$ for $0<\epsilon \leq r_{M^{n}}, \gamma$, i.e., $\operatorname{Sc}^{\mathrm{vol}_{n}}\left(M^{n}\right) \geq \kappa>0$.

Therefore, we have $S_{\frac{\kappa}{n-1}}^{n}<_{\text {vol }} S^{2}(\gamma) \times \mathbf{R}^{n-2}$ for all $\gamma>\sqrt{\frac{2}{n \kappa}}$. Here $S_{\frac{\kappa}{n-1}}^{n}$ is the Riemannian manifold $S^{n}$ with constant sectional curvature $\frac{\kappa}{n-1}$.

Remark 2.5. For a closed smooth Riemannian manifold $\left(M^{n}, g\right), \operatorname{Sc}^{\operatorname{vol}_{n}}\left(M^{n}\right) \geq 0$ implies $\mathrm{Sc}_{g} \geq 0$. Otherwise, there exists a point in $M^{n}$ such that the scalar curvature is negative, then the volume of small ball will be greater than the volume of the small ball in Euclidean space, which is a contradiction.

On the other hand, one can consider the case of the scalar-flat metric, i.e., $\mathrm{Sc}_{g} \equiv 0$. If $g$ is a strongly scalar-flat metric, meaning a metric with scalar curvature zero such that $M^{n}$ has no metric with positive scalar curvature, then $g$ is also Ricci flat according to Kazdan theorem above. Thus, we have

$$
\mathrm{dVol}_{g}\left(B_{r}(x)\right)=\operatorname{vol}_{E}\left(B_{r}\left(\mathbf{R}^{n}\right)\right)\left[1-\frac{\|\operatorname{Rie}(x)\|_{g}^{2}}{120(n+2)(n+4)} r^{4}+O\left(r^{6}\right)\right]
$$

for $B_{r}(x) \subset M^{n}$ as $r \rightarrow 0$ [14, Theorem 3.3]. Here Rie is the Riemannian tensor. Therefore, if $g$ is a not flat metric, then $M^{n}<_{\text {vol }} \mathbf{R}^{n}$. If $g$ is a flat metric, then $M^{n} \leq_{\text {vol }} \mathbf{R}^{n}$. Thus $\mathrm{Sc}_{g} \geq 0$ implies $\operatorname{Sc}^{\operatorname{vol}_{n}}\left(M^{n}\right) \geq 0$ for a strongly scalar-flat metric $g$.

However, $\mathrm{Sc}_{g} \geq 0$ may not imply $\operatorname{Sc}^{\operatorname{vol}_{n}}\left(M^{n}\right) \geq 0$. There are a lot of scalar-flat metrics but not strongly scalar flat metrics, i.e., $\mathrm{Sc}_{g} \equiv 0$ but not $\operatorname{Ricc}_{g} \neq 0$. For instance, the product metric on $S^{2}(1) \times \Sigma$, where $\Sigma$ is a closed hyperbolic surface, is the scalar-flat metric, but not the Ricci-flat metric. For those metrics, we have

$$
\mathrm{dVol}_{g}\left(B_{r}(x)\right)=\operatorname{vol}_{E}\left(B_{r}\left(\mathbf{R}^{n}\right)\right)\left[1+\frac{-3\|\operatorname{Rie}(x)\|_{g}^{2}+8\|\operatorname{Ricc}(x)\|_{g}^{2}}{360(n+2)(n+4)} r^{4}+O\left(r^{6}\right)\right]
$$

for $B_{r}(x) \subset M^{n}$ as $r \rightarrow 0$ [14, Theorem 3.3]. If $8\|\operatorname{Ricc}(x)\|_{g}^{2}>-3\|\operatorname{Rie}(x)\|_{g}^{2}$ for some point, then $\mathrm{Sc}_{g} \geq 0$ does not imply $\operatorname{Sc}^{\operatorname{vol}_{n}}\left(M^{n}\right) \geq 0$.

Theorem 2.6. Assume that the mm-space $\left(X^{n}, d, \mu\right)$ satisfies $n$-dimensional condition and the curvature-dimension condition $\mathrm{CD}(\kappa, n)$ for $\kappa \geq 0$ and $n \geq 2$, then $\left(X^{n}, d, \mu\right)$ satisfies $\operatorname{Sc}^{\operatorname{vol}_{n}}\left(X^{n}\right) \geq n \kappa$. 
Proof. In fact, one only needs the generalized Bishop-Gromov volume growth inequality, which is implied by the curvature-dimension of $X^{n}$ [38, Theorem 2.3].

(i) If $\kappa=0$, then

$$
\frac{\mu\left(B_{r}(x)\right)}{\mu\left(B_{R}(x)\right)} \geq\left(\frac{r}{R}\right)^{n}
$$

for all $0<r<R$. That is

$$
\frac{\mu\left(B_{r}(x)\right)}{\operatorname{vol}_{E}\left(B_{r}\left(\mathbf{R}^{n}\right)\right)}=\frac{\mu\left(B_{r}(x)\right)}{\alpha(n) r^{n}} \geq \frac{\mu\left(B_{R}(x)\right)}{\alpha(n) R^{n}}=\frac{\mu\left(B_{R}(x)\right)}{\operatorname{vol}_{E}\left(B_{R}\left(\mathbf{R}^{n}\right)\right)},
$$

where $\alpha(n)=\frac{\operatorname{vol}_{E}\left(B_{r}\left(\mathbf{R}^{n}\right)\right)}{r^{n}}$. Combining the $n$-dimensional condition,

$$
\lim _{r \rightarrow 0} \frac{\mu\left(B_{r}(x)\right)}{\operatorname{vol}_{E}\left(B_{r}\left(\mathbf{R}^{n}\right)\right)}=1
$$

that implies $\operatorname{Sc}^{\operatorname{vol}_{n}}(X) \geq 0$.

(ii) If $\kappa>0$, then

$$
\frac{\mu\left(B_{r}(x)\right)}{\mu\left(B_{R}(x)\right)} \geq \frac{\int_{0}^{r}\left[\sin \left(t \sqrt{\frac{\kappa}{(n-1)}}\right)\right]^{n-1} \mathrm{~d} t}{\int_{0}^{R}\left[\sin \left(t \sqrt{\frac{\kappa}{(n-1)}}\right)\right]^{n-1} \mathrm{~d} t}
$$

for all $0<r \leq R \leq \pi \sqrt{\frac{(n-1)}{\kappa}}$.

Since the scalar curvature of the product manifold $S^{2}(\gamma) \times \mathbf{R}^{n-2}$ is $n \kappa$, where $\gamma=\sqrt{\frac{2}{n \kappa}}$, then there exists $C_{1}, C_{2}>0$ such that

$$
1-\frac{n \kappa}{6(n+2)} r_{1}^{2}-C_{2} r_{1}^{4} \leq \operatorname{vol}_{S \times E}\left(B_{r_{1}}(y)\right):=\frac{\operatorname{vol}_{S \times E}\left(B_{r_{1}}(y)\right)}{\operatorname{vol}_{E}\left(B_{r_{1}}\left(\mathbf{R}^{n}\right)\right)} \leq 1-\frac{n \kappa}{6(n+2)} r_{1}^{2}+C_{2} r_{1}^{4},
$$

for $y \in S^{2}(\gamma) \times \mathbf{R}^{n-2}$ and $r_{1} \leq C_{1}$, where $C_{1}, C_{2}$ are decided by the product manifold $S^{2}(\gamma) \times$ $\mathbf{R}^{n-2}$.

Let

$$
\left.\mu \widetilde{\left(B_{r}(x)\right.}\right):=\frac{\mu\left(B_{r}(x)\right)}{\operatorname{vol}_{E}\left(B_{r}\left(\mathbf{R}^{n}\right)\right)}
$$

and

$$
f(r):=\frac{\int_{0}^{r}\left[\sin \left(t \sqrt{\frac{\kappa}{(n-1)}}\right)\right]^{n-1} \mathrm{~d} t}{\operatorname{vol}_{E}\left(B_{r}\left(\mathbf{R}^{n}\right)\right)},
$$

then the generalized Bishop-Gromov inequality can be re-formulated as

$$
\left.\left.\mu \widetilde{\left(B_{R}(x)\right.}\right) \leq \widetilde{\mu\left(B_{r}(x)\right.}\right) \frac{f(R)}{f(r)}
$$

for all $0<r<R \leq \pi \sqrt{\frac{(n-1)}{\kappa}}$. The asymptotic expansion of $f(r)$ is

$$
f(r)=\frac{\frac{1}{n} r^{n}\left[\frac{\kappa}{(n-1)}\right]^{\frac{(n-1)}{2}}-\frac{(n-1)}{6(n+2)} r^{n+2}\left[\frac{\kappa}{(n-1)}\right]^{\frac{n+1}{2}}+O\left(r^{n+4}\right)}{\operatorname{vol}_{E}\left(B_{r}\left(\mathbf{R}^{n}\right)\right)}
$$


as $r \rightarrow 0$. Thus, the asymptotic expansion of $\frac{f(R)}{f(r)}$ is

$$
\frac{f(R)}{f(r)}=\frac{1-\frac{n \kappa}{6(n+2)} R^{2}+O\left(R^{4}\right)}{1-\frac{n \kappa}{6(n+2)} r^{2}+O\left(r^{4}\right)}
$$

as $R \rightarrow 0, r \rightarrow 0$. The $n$-dimensional condition, $\left.\lim _{r \rightarrow 0} \mu \widetilde{\left(B_{r}(x)\right.}\right)=1$, implies that

$$
\left.\mu \widetilde{\left(B_{R}(x)\right.}\right) \leq 1-\frac{n \kappa}{6(n+2)} R^{2}+O\left(R^{4}\right)
$$

as $R \rightarrow 0$. Therefore, for any $\kappa^{\prime}$ with $0<\kappa^{\prime}<\kappa$, there exists $\epsilon_{\kappa^{\prime}}>0$ such that for any $0<R \leq \epsilon_{\kappa^{\prime}}$, we have

$$
\left.\mu \widetilde{\left(B_{R}(x)\right.}\right)<\operatorname{vol}_{S \times E}\left(B_{R}(y)\right)
$$

where $\operatorname{vol}_{S \times E}\left(B_{R}(y)\right)=\frac{\operatorname{vol}_{S \times E}\left(B_{R}(y)\right)}{\operatorname{vol}_{E}\left(B_{R}\left(\mathbf{R}^{n}\right)\right)}$ is defined as before, the balls $B_{R}(y)$ are in $S^{2}(\gamma) \times \mathbf{R}^{n-2}$ and $\gamma=\sqrt{\frac{2}{n \kappa^{\prime}}}$. That is $X^{n}<_{\text {vol }} S^{2}(\gamma) \times \mathbf{R}^{n-2}$, for all $\gamma>\sqrt{\frac{2}{n \kappa}}$, i.e., $\operatorname{Sc}^{\operatorname{vol}_{n}}\left(X^{n}\right) \geq n \kappa$.

In fact, one has the classical Bishop inequality by adding the $n$-dimensional condition to the generalized Bishop-Gromov volume growth inequality. It means that

- if $\kappa=0, \mu\left(B_{R}(x)\right) \leq \operatorname{vol}_{E}\left(B_{R}\left(\mathbf{R}^{n}\right)\right)$ for all $R>0$,

- if $\kappa>0, \mu\left(B_{R}(x)\right) \leq \operatorname{vol}_{S^{n}}\left(B_{R}\left(S_{\frac{\kappa}{n-1}}^{n}\right)\right)$ for $0<R \leq \pi \sqrt{\frac{(n-1)}{\kappa}}$.

In other words, if $\kappa=0, X^{n} \leq_{\mathrm{vol}} \mathbf{R}^{n}$. If $\kappa>0, X^{n} \leq_{\mathrm{vol}} S_{\frac{\kappa}{n-1}}^{n}$. We have $S_{\frac{\kappa}{n-1}}^{n}<_{\mathrm{vol}} S^{2}(\gamma) \times \mathbf{R}^{n-2}$ for all $\gamma>\sqrt{\frac{2}{n \kappa}}$. Then $X^{n}<_{\text {vol }} S^{2}(\gamma) \times \mathbf{R}^{n-2}$ for all $\gamma>\sqrt{\frac{2}{n \kappa}}$.

Thus, we also get $\mathrm{Sc}^{\mathrm{vol}_{n}}\left(X^{n}\right) \geq n \kappa$.

Remark 2.7. Hence the mm-space $\left(X^{n}, d, \mu\right)$ with $\operatorname{Sc}^{\operatorname{vol}_{n}}\left(X^{n}\right) \geq n \kappa$ includes the mm-spaces that satisfies $n$-dimensional condition and the generalized Bishop-Gromov volume growth inequality as stated in the proof, e.g., the mm-spaces with the Riemannian curvature condition $\operatorname{RCD}(\kappa, n)$ [2] or with the measure concentration property $\operatorname{MCP}(\kappa, n)$ [29].

Question 2.8. Let $\mathrm{Al}^{n}(1)$ be an orientable compact n-dimensional Aleksandrov space with curvature $\geq 1$, then do all continuous maps $\phi$ from $\mathrm{Al}^{n}(1)$ to the sphere $S^{n}$ with standard metric of non-zero degree satisfy $\operatorname{Lip}(\phi) \geq C(n)$ ? Here $\operatorname{Lip}(\phi)$ is the Lipschitz constant of $\phi, \phi$ maps the boundary of $\mathrm{Al}^{n}(1)$ to a point in $S^{n}$ and $C(n)$ is a constant depending only on the dimension $n$.

Question 2.9. Assume the compact mm-space $(X, d, \mu)$ satisfies the curvature-dimension condition $\mathrm{CD}(n-1, n), n$-dimensional condition and the covering dimension is also $n$, then do all continuous maps $\phi$ from $(X, d, \mu)$ to the sphere $S^{n}$ with standard metric, where $\phi$ is non-trivial in the homotopy class of maps, satisfy $\operatorname{Lip}(\phi) \geq C_{1}(n)$, where $C_{1}(n)$ is a constant depending only on $n$ ?

Remark 2.10. The questions above are inspired by Gromov's spherical Lipschitz bounded theorem in [19, Section 3] and the results above. The finite covering dimension is equal to the cohomological dimension over integer ring $\mathbb{Z}$ for the compact metric space according to the Alexandrov theorem. The best constant of $C(n)$ and $C_{1}(n)$ would be 1 if both questions have positive answers. 
Proposition 2.11 (quadratic scaling). Assume the compact mm-space $\left(X^{n}, d, \mu\right)$ satisfies $\mathrm{Sc}^{\operatorname{vol}_{n}}\left(X^{n}\right) \geq \kappa>0$, then $\mathrm{Sc}^{\operatorname{vol}_{n}}\left(\lambda X^{n}\right) \geq \lambda^{-2} \kappa>0$ and $r_{\lambda X^{n}}=\lambda r_{X^{n}}$ for all $\lambda>0$, where $\lambda X^{n}:=\left(X^{n}, \lambda \cdot d, \lambda^{n} \cdot \mu\right)$.

Proof. First, we will show that the $n$-dimensional condition is stable under scaling. Let $d^{\prime}:=$ $\lambda \cdot d, \mu^{\prime}:=\lambda^{n} \cdot \mu, B_{r}^{\prime}(x)$ be an $r$-ball in the $\left(X^{n}, d^{\prime}\right)$, and $B_{r}(x)$ be an $r$-ball in the $\left(X^{n}, d\right)$, then $B_{r}^{\prime}(x)=B_{\frac{r}{\lambda}}(x)$ as the subset in the $X^{n}$. One has

$$
\lim _{r \rightarrow 0} \frac{\mu^{\prime}\left(B_{r}^{\prime}(x)\right)}{\operatorname{vol}_{E}\left(B_{r}\left(\mathbf{R}^{n}\right)\right)}=\lim _{r \rightarrow 0} \frac{\mu^{\prime}\left(B_{\frac{r}{\lambda}}(x)\right)}{\operatorname{vol}_{E}\left(B_{r}\left(\mathbf{R}^{n}\right)\right)}=\lim _{r \rightarrow 0} \frac{\lambda^{n} \cdot \mu\left(B_{\frac{r}{\lambda}}(x)\right)}{\lambda^{n} \cdot \operatorname{vol}_{E}\left(B_{\frac{r}{\lambda}}\left(\mathbf{R}^{n}\right)\right)}=1,
$$

then $\lambda X^{n}$ satisfies the $n$-dimensional condition.

Since $\lambda \cdot\left(S^{2}(\gamma) \times \mathbf{R}^{n-2}\right)=\lambda \cdot S^{2}(\gamma) \times \lambda \cdot \mathbf{R}^{n-2}=S^{2}(\lambda \gamma) \times \lambda \cdot \mathbf{R}^{n-2}$, we have

$$
\lambda \cdot X^{n}<_{\text {vol }} \lambda \cdot\left(S^{2}(\gamma) \times \mathbf{R}^{n-2}\right)=S^{2}(\lambda \gamma) \times \lambda \cdot \mathbf{R}^{n-2}
$$

for all $\lambda \gamma>\sqrt{\frac{2}{\lambda \kappa}}$ and $0<\epsilon \leq \lambda r_{X^{n}}$. That means $\operatorname{Sc}^{\operatorname{vol}_{n}}\left(\lambda X^{n}\right) \geq \lambda^{-2} \kappa>0$ and $r_{\lambda \cdot X^{n}}=$ $\lambda r_{X^{n}}$.

We also have $\operatorname{Sc}^{\operatorname{vol}_{n}}\left(\lambda X^{n}\right) \geq 0(>0)$, if $\mathrm{Sc}^{\operatorname{vol}_{n}}\left(X^{n}\right) \geq 0(>0)$.

Remark 2.12. Since the $n$-dimensional condition and definition of $n$-volumic scalar curvature is locally defined, we have the following construction.

- Global to local: Let the locally compact mm-space $\left(X^{n}, d, \mu\right)$ satisfy $\operatorname{Sc}^{\operatorname{vol}_{n}}\left(X^{n}\right) \geq \kappa \geq 0$ and $Y^{n} \subset X$ be an open subset. Then, if $\left(Y^{n}, d_{Y}\right)$ is a complete length space, $\left(Y^{n}, d_{Y}, \mu\llcorner Y)\right.$ satisfies $\operatorname{Sc}^{\operatorname{vol}_{n}}\left(Y^{n}\right) \geq \kappa \geq 0$ and $r_{Y^{n}}=r_{X^{n}}$. Where $d_{Y}$ is the induced metric of $d$ and $\mu\llcorner Y$ is the restriction operator, namely, $\mu\left\llcorner Y(A):=\mu\left(Y^{n} \cap A\right)\right.$ for $A \subset X^{n}$.

- Local to global: Let $\left\{Y_{i}^{n}\right\}_{i \in I}$ be a finite open cover of a locally compact mm-space $\left(X^{n}, d, \mu\right)$. Assume that $\left(Y_{i}^{n}, d_{Y_{i}}\right)$ is a complete length space and $\left(Y_{i}^{n}, d_{Y_{i}}, \mu\left\llcorner Y_{i}\right)\right.$ satisfies $\operatorname{Sc}^{\operatorname{vol}_{n}}\left(Y_{i}^{n}\right) \geq \kappa \geq 0$, then $\left(X^{n}, d, \mu\right)$ satisfies $\operatorname{Sc}^{\operatorname{vol}_{n}}\left(X^{n}\right) \geq \kappa \geq 0$ and $r_{X^{n}}$ can be chosen as a partition of unity of the functions $\left\{r_{Y_{i}^{n}}\right\}_{i \in I}$.

Question 2.13. Assume that $\mathrm{Sc}^{\operatorname{vol}_{n_{1}}}\left(X_{1}^{n_{1}}\right) \geq \kappa_{1}(\geq 0)$ for the compact mm-space $\left(X_{1}^{n_{1}}, d_{1}, \mu_{1}\right)$ and $\mathrm{Sc}^{\mathrm{vol}_{n_{2}}}\left(X_{2}^{n_{2}}\right) \geq \kappa_{2}(\geq 0)$ for the compact mm-space $\left(X_{2}^{n_{2}}, d_{2}, \mu_{2}\right)$, then do we have

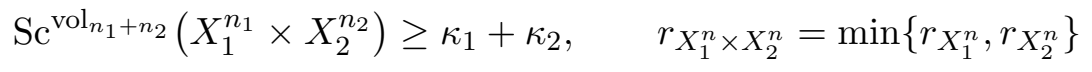

for $\left(X_{1}^{n_{1}} \times X_{2}^{n_{2}}, d_{3}, \mu_{3}\right)$ ? Here $X_{1}^{n_{1}} \times X_{2}^{n_{2}}$ is endowed with the measure $\mu_{3}:=\mu_{1} \otimes \mu_{2}$ and with the Pythagorean product metric $d_{3}:=\sqrt{d_{1}^{2}+d_{2}^{2}}$.

\section{3 smGH-convergence}

Let $\left\{\mu_{n}\right\}_{n \in \mathbf{N}}$ and $\mu$ be Borel measures on the space $X$, then the sequence $\left\{\mu_{n}\right\}_{n \in \mathbf{N}}$ is said to converge strongly (also called setwise convergence in other literature ) to a limit $\mu$ if $\lim _{n \rightarrow \infty} \mu_{n}(\mathcal{A})=$ $\mu(\mathcal{A})$ for every $\mathcal{A}$ in the Borel $\sigma$-algebra.

A map $f: X \rightarrow Y$ is called an $\epsilon$-isometry between compact metric spaces $X$ and $Y$, if $\left|d_{X}(a, b)-d_{Y}(f(a), f(b))\right| \leq \epsilon$ for all $a, b \in X$ and it is almost surjective, i.e., for every $y \in Y$, there exists an $x \in X$ such that $d_{Y}(f(x), y) \leq \epsilon$.

In fact, if $f$ is an $\epsilon$-isometry $X \rightarrow Y$, then there is a $(4 \epsilon)$-isometry $f^{\prime}: Y \rightarrow X$ such that for all $x \in X, y \in Y, d_{X}\left(f^{\prime} \circ f(x), x\right) \leq 3 \epsilon, d_{Y}\left(f \circ f^{\prime}(y), y\right) \leq \epsilon$. 
Definition 3.1 (smGH-convergence). Let $\left(X_{i}, d_{i}, \mu_{i}\right)_{i \in \mathbf{N}}$ and $(X, d, \mu)$ be compact mm-spaces. $X_{i}$ converges to $X$ in the strongly measured Gromov-Hausdorff topology (smGH-convergence) if there are measurable $\epsilon_{i}$-isometries $f_{i}: X_{i} \rightarrow X$ such that $\epsilon_{i} \rightarrow 0$ and $f_{i *} \mu_{i} \rightarrow \mu$ in the strong topology of measures as $i \rightarrow \infty$.

If the spaces $\left(X_{i}, d_{n}, \mu_{i}, p_{i}\right)_{i \in \mathbf{N}}$ and $(X, d, \mu, p)$ are locally compact pointed mm-spaces, it is said that $X_{i}$ converges to $X$ in the pointed strongly measured Gromov-Hausdorff topology (psmGH-convergence) if there are sequences $r_{i} \rightarrow \infty, \epsilon_{i} \rightarrow 0$, and measurable pointed $\epsilon_{i}$-isometries $f_{i}: B_{r_{i}}\left(p_{i}\right) \rightarrow B_{r_{i}}(p)$, such that $f_{i *} \mu_{i} \rightarrow \mu$, where the convergence is strong convergence.

Remark 3.2. Let $\left(X_{i}, d_{i}, \mu_{i}\right)_{i \in \mathbf{N}}$ converge to $(X, d, \mu)$ in the measured Gromov-Hausdorff topology, then there are measurable $\epsilon_{i}$-isometries $f_{i}: X_{i} \rightarrow X$ such that $f_{i *} \mu_{i}$ weakly converges to $\mu$. If there is a Borel measure $\nu$ on $X$ such that $\sup _{i} f_{i *} \mu_{i} \leq \nu$, i.e., $\sup _{i} f_{i *} \mu_{i}(\mathcal{A}) \leq \nu(\mathcal{A})$ for every $\mathcal{A}$ in the Borel $\sigma$-algebra on $X$, then $X_{i}$ smGH-converges to $X$ (see [26, Lemma 4.1]).

Remark 3.3. The $n$-dimensional condition is not preserved by the measured Gromov-Hausdorff convergence as the following example shows. Let $\left\{a_{i} S^{2}:=\left(S^{2}, a_{i} d_{S}\right)\right\}\left(a_{i} \in(0,1)\right)$ be a sequence of space, then the limit of $a_{i} S^{2}$ under the measured Gromov-Hausdorff convergence is a point when $a_{i}$ goes to 0 . The limit exists as the Ricci curvature of $a_{i} S^{2}$ is bounded below by 1 .

Remark 3.4. The $n$-dimensional condition is not preserved by the smGH-convergence since the limits of $\lim _{r \rightarrow 0} \lim _{i \rightarrow \infty} \frac{\mu_{i}\left(B_{r}(x)\right)}{\operatorname{vol}_{E}\left(B_{r}\left(\mathbf{R}^{n}\right)\right)}$ may not be commutative for some mm-spaces $\left(X, d, \mu_{i}\right)$. Assume the total variation distance of the measures goes to 0 as $i \rightarrow \infty$, i.e.,

$$
d_{T V}\left(\mu_{i}, \mu\right):=\sup _{\mathcal{A}}\left|\mu_{i}(\mathcal{A})-\mu(\mathcal{A})\right| \rightarrow 0,
$$

where $\mathcal{A}$ runs over the Borel $\sigma$-algebra of $X$, then the limits are commutative.

One can also define the total variation Gromov-Hausdorff convergence (tvGH-convergence) for mm-spaces by replacing the strong topology with the topology induced by the total variation distance in definition of smGH-convergence. Then tvGH-convergence implies smGH-convergence and the $n$-dimensional condition is preserved by tvGH-convergence.

Theorem 3.5 (stability). If compact mm-spaces $\left(X_{i}^{n}, d_{i}, \mu_{i}\right)$ with $\mathrm{Sc}^{\operatorname{vol}_{n}}\left(X_{i}^{n}\right) \geq \kappa \geq 0$, SCradius $r_{X_{i}^{n}} \geq R>0$, and $\left(X_{i}^{n}, d_{i}, \mu_{i}\right)$ smGH-converge to the compact mm-space $\left(X^{n}, d, \mu\right)$ with $n$-dimensional condition, then $X^{n}$ also satisfies $\operatorname{Sc}^{\operatorname{vol}_{n}}\left(X^{n}\right) \geq \kappa$ and the $S C$-radius $r_{X^{n}} \geq R$.

Proof. Fix an $x \in X^{n}$ and let $B_{r}(x)$ be the small $r$-ball on $X^{n}$ where $r<R$, then there exists $x_{i} \in X_{i}^{n}$ such that $f_{i}^{-1}\left(B_{r}(x)\right) \subset B_{r+4 \epsilon_{i}}\left(x_{i}\right)$ where $B_{r+4 \epsilon_{i}}\left(x_{i}\right) \subset X_{i}^{n}$ and $r+4 \epsilon_{i} \leq R$. Thus, $f_{i *} \mu_{i}\left(B_{r}(x)\right) \leq \mu_{i}\left(B_{r+4 \epsilon_{i}}\left(x_{i}\right)\right)$.

- For $\kappa=0$, since $\mathrm{Sc}^{\operatorname{vol}_{n}}\left(X_{i}^{n}\right) \geq 0$ and SC-radius $\geq R>0$, then $\mu_{i}\left(B_{r}\left(x_{i}\right)\right) \leq \operatorname{vol}_{E}\left(B_{r}\left(\mathbf{R}^{n}\right)\right)$ for all $0<r \leq R$ and all $i$. Therefore, $f_{i *} \mu_{i}\left(B_{r}(x)\right)<\mu_{i}\left(B_{r+4 \epsilon_{i}}\left(x_{i}\right)\right) \leq \operatorname{vol}_{E}\left(B_{r+4 \epsilon_{i}}\left(\mathbf{R}^{n}\right)\right)$ for $r+4 \epsilon_{i} \leq R$. Since $\epsilon_{i}$ that is not related to $r$ can be arbitrarily small, then $\mu\left(B_{r}(x)\right) \leq$ $\operatorname{vol}_{E}\left(B_{r}\left(\mathbf{R}^{n}\right)\right)$.

- For $\kappa>0$, we have $\mu_{i}\left(B_{r}\left(x_{i}\right)\right)<\operatorname{vol}_{S \times E}\left(B_{r}\left(S^{2}(\gamma) \times \mathbf{R}^{n-2}\right)\right)$ for all $0<r \leq R$, all $i$, and $\gamma>\sqrt{\frac{2}{\kappa}}$. Thus, $f_{i *} \mu_{i}\left(B_{r}(x)\right)<\mu_{i}\left(B_{r+4 \epsilon_{i}}\left(x_{i}\right)\right)<\operatorname{vol}_{S \times E}\left(B_{r+4 \epsilon_{i}}\left(S^{2}(\gamma) \times \mathbf{R}^{n-2}\right)\right)$ for $r+4 \epsilon_{i} \leq R$. Since $\epsilon_{i}$ that is not related to $r$ can be arbitrarily small, then $\mu\left(B_{r}(x)\right) \leq$ $\operatorname{vol}_{S \times E}\left(B_{r}\left(S^{2}(\gamma) \times \mathbf{R}^{n-2}\right)\right)$ for $\gamma>\sqrt{\frac{2}{\kappa}}$. Thus, $\mu\left(B_{r}(x)\right)<\operatorname{vol}_{S \times E}\left(B_{r}\left(S^{2}\left(\sqrt{\frac{2}{\kappa+\epsilon^{\prime}}}\right) \times\right.\right.$ $\left.\mathbf{R}^{n-2}\right)$ ), where $0<\epsilon^{\prime}$ is independence on $r$ and $\epsilon^{\prime}$ can as small as we want. Therefore, we have $\operatorname{Sc}^{\operatorname{vol}_{n}}\left(X^{n}\right) \geq \kappa$. 
Definition 3.6 (tangent space). The mm-space $\left(Y, d_{Y}, \mu_{Y}, o\right)$ is a tangent space of $\left(X^{n}, d, \mu\right)$ at $p \in X^{n}$ if there exists a sequence $\lambda_{i} \rightarrow \infty$ such that $\left(X^{n}, \lambda_{i} \cdot d, \lambda_{i}^{n} \cdot \mu, p\right)$ psmGH-converges to $\left(Y, d_{Y}, \mu_{Y}, o\right)$ as $\lambda_{i} \rightarrow \infty$.

Therefore, $\left(Y, d_{Y}, \mu_{Y}, o\right)$ also satisfies the $n$-dimensional condition and can be written as $Y^{n}$.

Corollary 3.7. Assume the compact mm-space $\left(X^{n}, d, \mu\right)$ with $\operatorname{Sc}^{\operatorname{vol}_{n}}\left(X^{n}\right) \geq \kappa \geq 0$ and the tangent space $\left(Y^{n}, d_{Y}, \mu_{Y}, o\right)$ of $X^{n}$ exists at the point $p$, then $\left(Y^{n}, d_{Y}, \mu_{Y}, o\right)$ satisfies $\operatorname{Sc}^{\operatorname{vol}_{n}}\left(Y^{n}\right)$ $\geq 0$ and the $S C$-radius $\geq r_{X^{n}}$.

Proof. Since the $n$-volumic scalar curvature has the quadratic scaling property, i.e., $\operatorname{Sc}^{\mathrm{vol}_{n}}\left(\lambda X^{n}\right)$ $\geq \lambda^{-2} \kappa \geq 0$ and $r_{\lambda X^{n}}=\lambda r_{X^{n}}$ for all $\lambda>0$, where $\lambda X^{n}:=\left(X^{n}, \lambda \cdot d, \lambda^{n} \cdot \mu\right)$, then $\operatorname{Sc}^{\operatorname{vol}_{n}}\left(Y^{n}\right) \geq 0$ is implied by the stability theorem.

The mm-spaces with $\mathrm{Sc}^{\mathrm{vol}_{n}} \geq 0$ includes some of the Finsler manifolds, for instance, $\mathbf{R}^{n}$ equipped with any norm and with the Lebesgue measure satisfies $\mathrm{Sc}^{\mathrm{vol}_{n}} \geq 0$ and any smooth compact Finsler manifold is a $\mathrm{CD}(\kappa, n)$ space for appropriate finite $\kappa$ and $n$ [30]. It is well-known that Gigli's infinitesimally Hilbertian [12] can be seen as the Riemannian condition in $\operatorname{RCD}(\kappa, n)$ space. Thus, infinitesimally Hilbertian can also be used as a Riemannian condition in the mmspaces with $\mathrm{Sc}^{\mathrm{vol}_{n}} \geq 0$.

Definition $3.8\left(\operatorname{RSC}(\kappa, n)\right.$ space). The compact mm-space $\left(X^{n}, d, \mu\right)$ with the $n$-dimensional condition is a Riemannian $n$-volumic scalar curvature $\geq \kappa$ space $(\operatorname{RSC}(\kappa, n)$ space) if it is infinitesimally Hilbertian and satisfies the $\operatorname{Sc}^{\operatorname{vol}_{n}}\left(X^{n}\right) \geq \kappa \geq 0$.

Note that any finite-dimensional Alexandrov spaces with curvature bounded below are infinitesimally Hilbertian. Then

$$
\mathrm{Al}^{n}(\kappa) \Rightarrow \operatorname{RCD}((n-1) \kappa, n) \Rightarrow \operatorname{RSC}((n(n-1) \kappa, n)
$$

on $\left(X^{n}, d, \mathcal{H}^{n}\right)$, where the measure $\mathcal{H}^{n}$ is the $n$-dimensional Hausdorff measure that satisfies the $n$-dimensional condition.

Question 3.9. Are $\operatorname{RSC}(\kappa, n)$ spaces stable under tvGH-convergence?

Remark 3.10 (convergence of compact mm-spaces). For the compact metric measure spaces with probability measures, one can consider mGH-convergence, Gromov-Prokhorov convergence, Gromov-Hausdorff-Prokhorov convergence, Gromov-Wasserstein convergence, GromovHausdorff-Wasserstein convergence, Gromov's ㅁ-convergence, Sturm's $\mathbb{D}$-convergence [39, Section 27], and Gromov-Hausdorff-vague convergence [3]. smGH-convergence implies those convergences for compact metric measure spaces with probability measures, since the measures converge strongly in smGH-convergence and converge weakly in other situations.

Note that mm-spaces with infinitesimally Hilbertian are not stable under mGH-convergence [12]. It is not clear if the infinitesimally Hilbertian are preserved under smGH-convergence or tvGH-convergence.

\section{Smooth mm-space with $\mathrm{Sc}_{\alpha, \beta}>0$}

Let the smooth metric measure space $\left(M^{n}, g, \mathrm{e}^{-f} \mathrm{dVol}_{g}\right)$ (also known as the weighted Riemannian manifold in some references), where $f$ is a $C^{2}$-function on $M^{n}, g$ is a $C^{2}$-Riemannian metric and $n \geq 2$, satisfy the curvature-dimension condition $\mathrm{CD}(\kappa, n)$ for $\kappa \geq 0$, then $M^{n}$ also satisfies $\mathrm{Sc}^{\operatorname{vol}_{n}}\left(M^{n}\right) \geq n \kappa$. 
Motivated by the importance of the Ricci Bakry-Emery curvature, i.e.,

$$
\operatorname{Ric}_{f}^{M}=\operatorname{Ricc}+\operatorname{Hess}(f)
$$

the weighted sectional curvature of smooth mm-space was proposed and discussed in [40]. On the other hand, Perelman defined and used the P-scalar curvature in his $\mathcal{F}$-functional in [31, Section 1]. Inspired by the P-scalar curvature, i.e., $\mathrm{Sc}_{g}+2 \triangle_{g} f-\|\nabla g f\|_{g}^{2}$, we propose another scalar curvature on the smooth mm-space.

Definition 4.1 (weighted scalar curvature $\mathrm{Sc}_{\alpha, \beta}$ ). The weighted scalar curvature $\mathrm{Sc}_{\alpha, \beta}$ on the smooth mm-space $\left(M^{n}, g, \mathrm{e}^{-f} \mathrm{dVol}_{g}\right)$ is defined by

$$
\mathrm{Sc}_{\alpha, \beta}:=\mathrm{Sc}_{g}+\alpha \triangle_{g} f-\beta\|\nabla g f\|_{g}^{2} .
$$

Note that the Laplacian $\triangle_{g}$ here is the trace of the Hessian and $\operatorname{Sc}^{\operatorname{vol}_{n}}\left(M^{n}\right) \geq \kappa \geq 0$ is equivalent to $\mathrm{Sc}_{\alpha, \beta} \geq \kappa \geq 0$ for $\alpha=3$ and $\beta=3$ (see [33, Theorem 8] or the proof of Corollary 4.10 below).

\section{Example 4.2.}

1. For $\alpha=\frac{2(n-1)}{n}$ and $\beta=\frac{(n-1)(n-2)}{n^{2}}$, the $\operatorname{Sc}_{\frac{2(n-1)}{n}, \frac{(n-1)(n-2)}{n^{2}}}$ is the Chang-Gursky-Yang's conformally invariant scalar curvature for the smooth mm-space [7]. That means for a $C^{2}-$ smooth function $w$ on $M^{n}$, one has

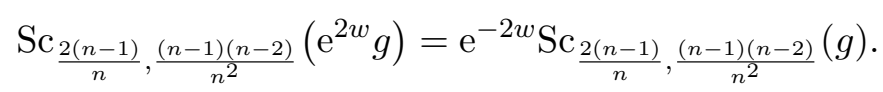

2. For $\alpha=2$ and $\beta=\frac{m+1}{m}$, where $m \in \mathbb{N} \cup\{0, \infty\}$, the $\mathrm{Sc}_{2, \frac{m+1}{m}}$ is Case's weighted scalar curvature and Case also defined and studied the weighted Yamabe constants in [6]. Case's weighted scalar curvature is the classical scalar curvature if $m=0$. If $m=\infty$, then it is Perelman's $P$-scalar curvature.

Note that the results in this paper are new for those examples.

\subsection{Spin manifold and $\mathrm{Sc}_{\alpha, \beta}>0$}

For an orientable closed surface with density $\left(\Sigma, g, \mathrm{e}^{-f} \mathrm{dVol}_{g}\right)$ with $\mathrm{Sc}_{\alpha, \beta}>0$ and $\beta \geq 0$, then the inequality,

$$
0<\int_{\Sigma} \mathrm{Sc}_{\alpha, \beta} \mathrm{dVol}_{g}=\int_{\Sigma}\left(\mathrm{Sc}_{g}+\alpha \triangle_{g} f-\beta\left\|\nabla_{g} f\right\|_{g}^{2}\right) \mathrm{dVol}_{g}=4 \pi \chi(\Sigma)-\beta \int_{\Sigma}\|\nabla g f\|_{g}^{2} \mathrm{dVol}_{g}
$$

implies that $\chi(\Sigma)>0$. Thus, $\Sigma$ is a 2 -sphere.

The following proposition of vanishing harmonic spinors is owed to Perelman essentially and the proof is borrowed from [1, Proposition 1].

Proposition 4.3 (vanishing harmonic spinors). Assume the smooth $m m$-space $\left(M^{n}, g, \mathrm{e}^{-f} \mathrm{dVol}_{g}\right)$ is closed and spin. If $\alpha \in \mathbb{R}, \beta \geq \frac{|\alpha|^{2}}{4}$ and $\mathrm{Sc}_{\alpha, \beta}>0$, then the harmonic spinor of $M^{n}$ vanishes.

Proof. Let $\psi$ be a harmonic spinor, though the Schrödinger-Lichnerowicz-Weitzenboeck formula

$$
\mathbb{D}^{2}=\nabla^{*} \nabla+\frac{1}{4} \mathrm{Sc}_{g}
$$


one has

$$
\begin{aligned}
0 & =\int_{M}\left[\left\|\nabla_{g} \psi\right\|_{g}^{2}+\frac{1}{4}\left(\operatorname{Sc}_{\alpha, \beta}-\alpha \triangle_{g} f+\beta\left\|\nabla_{g} f\right\|_{g}^{2}\right)\|\psi\|_{g}^{2}\right] \mathrm{dVol}_{g} \\
& =\int_{M}\left[\left\|\nabla_{g} \psi\right\|_{g}^{2}+\left(\frac{1}{4} \operatorname{Sc}_{\alpha, \beta}+\frac{\beta}{4}\left\|\nabla_{g} f\right\|_{g}^{2}\right)\|\psi\|_{g}^{2}+\frac{\alpha}{4}\left\langle\nabla_{g} f, \nabla g\|\psi\|_{g}^{2}\right\rangle_{g}\right] \mathrm{dVol}_{g} .
\end{aligned}
$$

Then one gets

$$
\begin{aligned}
\frac{|\alpha|}{4}\left|\left\langle\nabla_{g} f, \nabla_{g}\|\psi\|_{g}^{2}\right\rangle_{g}\right| & \leq \frac{|\alpha|}{4}\left(c\left\|\nabla_{g} f\right\|_{g}\|\psi\|_{g} \times 2 c^{-1}\left\|\nabla_{g} \psi\right\|_{g}\right) \\
& \leq \frac{|\alpha| c^{2}}{8}\left\|\nabla_{g} f\right\|_{g}^{2}\|\psi\|_{g}^{2}+\frac{c^{-2}|\alpha|}{2}\left\|\nabla_{g} \psi\right\|_{g}^{2} .
\end{aligned}
$$

Therefore,

$$
0 \geq \int_{M}\left[\left(1-\frac{c^{-2}|\alpha|}{2}\right)\left\|\nabla_{g} \psi\right\|_{g}^{2}+\frac{2 \beta-c^{2}|\alpha|}{8}\left\|\nabla_{g} f\right\|_{g}^{2}\|\psi\|_{g}^{2}+\frac{1}{4} \operatorname{Sc}_{\alpha, \beta}\|\psi\|_{g}^{2}\right] \mathrm{dVol}_{g}
$$

where $c \neq 0$. If $c^{-2}|\alpha| \leq 2, \beta \geq \frac{c^{2}|\alpha|}{2}$ and $\operatorname{Sc}_{\alpha, \beta}>0$, then $\psi=0$. So the conditions $\alpha \in \mathbb{R}$ and $\beta \geq \frac{|\alpha|^{2}}{4}$ are needed

$$
\begin{aligned}
\frac{|\alpha|}{4}\left|\left\langle\nabla_{g} f, \nabla_{g}\|\psi\|_{g}^{2}\right\rangle_{g}\right| & \leq \frac{|\alpha|}{4}\left(\left\|\nabla_{g} f\right\|_{g}\|\psi\|_{g} \times 2\left\|\nabla_{g} \psi\right\|_{g}\right) \\
& =\frac{|\alpha|}{2}\left(c_{1}\left\|\nabla_{g} f\right\|_{g}\|\psi\|_{g} \times c_{1}^{-1}\left\|\nabla_{g} \psi\right\|_{g}\right) \\
& \leq \frac{|\alpha|}{4}\left(c_{1}^{2}\left\|\nabla_{g} f\right\|_{g}^{2}\|\psi\|_{g}^{2}+c_{1}^{-2}\left\|\nabla_{g} \psi\right\|_{g}^{2}\right) .
\end{aligned}
$$

Thus,

$$
0 \geq \int_{M}\left[\left(1-\frac{c_{1}^{-2}|\alpha|}{4}\right)\left\|\nabla_{g} \psi\right\|_{g}^{2}+\frac{\beta-c_{1}^{2}|\alpha|}{4}\left\|\nabla_{g} f\right\|_{g}^{2}\|\psi\|_{g}^{2}+\frac{1}{4} \operatorname{Sc}_{\alpha, \beta}\|\psi\|_{g}^{2}\right] \mathrm{dVol}_{g}
$$

where $c_{1} \neq 0$. If $c_{1}^{-2}|\alpha| \leq 4, \beta \geq c_{1}^{2}|\alpha|$ and $\mathrm{Sc}_{\alpha, \beta}>0$, then $\psi=0$. Also the conditions $\alpha \in \mathbb{R}$ and $\beta \geq \frac{|\alpha|^{2}}{4}$ are needed.

The following 3 corollaries come from the proposition of vanishing of harmonic spinors.

Corollary 4.4. Assume the smooth mm-space $\left(M^{n}, g, \mathrm{e}^{-f} \mathrm{dVol}_{g}\right)$ is closed and spin. If $\alpha \in \mathbb{R}$, $\beta \geq \frac{|\alpha|^{2}}{4}$ and $\mathrm{Sc}_{\alpha, \beta}>0$, then the $\widehat{A}$-genus and the Rosenberg index of $M^{n}$ vanish.

Proof. Since the $C^{*}\left(\pi_{1}\left(M^{n}\right)\right)$-bundle in the construction of the Rosenberg index [35] is flat, there are no correction terms due to curvature of the bundle. Then the Schrödinger-Lichnerowicz-Weitzenboeck formula and the argument in the proof of vanishing harmonic spinors can be applied without change.

Corollary 4.5. Assume that $M^{n}$ is a closed spin n-manifold and $f$ is a smooth function on $M^{n}$. If one of the following conditions is met,

(1) $N \subset M^{n}$ is a codimension one closed connected submanifold with trivial normal bundle, the inclusion of fundamental groups $\pi_{1}\left(N^{n-1}\right) \rightarrow \pi_{1}\left(M^{n}\right)$ is injective and the Rosenberg index of $N$ does not vanish, or 
(2) $N \subset M^{n}$ is a codimension two closed connected submanifold with trivial normal bundle, $\pi_{2}\left(M^{n}\right)=0$, the inclusion of fundamental groups $\pi_{1}\left(N^{n-1}\right) \rightarrow \pi_{1}\left(M^{n}\right)$ is injective and the Rosenberg index of $N$ does not vanish, or

(3) $N=N_{1} \cap \cdots \cap N_{k}$, where $N_{1} \cdots N_{k} \subset M$ are closed submanifolds that intersect mutually transversely and have trivial normal bundles. Suppose that the codimension of $N_{i}$ is at most two for all $i \in\{1 \dot{k}\}$ and $\pi_{2}(N) \rightarrow \pi_{2}(M)$ is surjective and $\hat{A}(N) \neq 0$,

then $M^{n}$ does not admit a Riemnannian metric $g$ such that the smooth mm-space $\left(M^{n}, g\right.$, $\mathrm{e}^{-f} \operatorname{dvol}_{g}$ ) satisfies $\mathrm{Sc}_{\alpha, \beta}>0$ for the dimension $n \geq 3, \alpha \in \mathbb{R}$ and $\beta \geq \frac{|\alpha|^{2}}{4}$.

Proof. The results in the [22, Theorem 1.1] and [42, Theorem 1.9] can be applied to show that the Rosenberg index of $M^{n}$ does not vanish and Corollary 4.4 implies the theorem.

Let $\mathcal{R}_{f}\left(M^{n}\right):=\{(g, f)\}$ be the space of densities, where $g$ is a smooth Riemannian metric on $M^{n}$ and $f$ is a smooth function on $M^{n}$ and $\mathcal{R}_{f}^{+}\left(M^{n}\right) \subset \mathcal{R}_{f}\left(M^{n}\right)$ is the subspace of densities such that the smooth mm-space $\left(M^{n}, g, \mathrm{e}^{-f} \mathrm{dvol}_{g}\right)$ satisfies $\mathrm{Sc}_{\alpha, \beta}>0$. Furthermore, let $\mathcal{R}_{f}^{+}\left(M^{n}\right)$ be endowed with the smooth topology.

Corollary 4.6. Assume $M^{n}$ is a closed spin $n$-manifold, $n \geq 3, \alpha \in \mathbb{R}$ and $\beta \geq \frac{|\alpha|^{2}}{4}$ and $\mathcal{R}_{f}^{+}\left(M^{n}\right) \neq \varnothing$, then there exists a homomorphism

$$
A_{m-1}: \pi_{m-1}\left(\mathcal{R}_{f}^{+}\left(M^{n}\right)\right) \rightarrow K O_{n+m}
$$

such that

- $A_{0} \neq 0$, if $n \equiv 0,1(\bmod 8)$,

- $A_{1} \neq 0$, if $n \equiv-1,0(\bmod 8)$,

- $A_{8 j+1-n} \neq 0$, if $n \geq 7$ and $8 j-n \geq 0$.

Proof. Since the results in the [23, Section 4.4] and [9] depend on the existence of exotic spheres with non-vanishing $\alpha$-invariant. Let $\phi: M^{n} \rightarrow M^{n}$ be a diffeomorphism of $M^{n}$ and $(g, f) \in \mathcal{R}_{f}^{+}\left(M^{n}\right)$, then $\left(\phi^{*} g, f \circ \phi\right)$ is also in $\mathcal{R}_{f}^{+}\left(M^{n}\right)$. Combining it with Proposition 4.3 shows that Hitchin's construction of the map $A$ [23, Proposition 4.6] can be applied to the case of $\mathcal{R}_{f}^{+}\left(M^{n}\right)$ and then we can finish the proof with the arguments in [23, Section 4.4] and [9, Section 2.5].

\subsection{Conformal to PSC-metrics}

Proposition 4.7 (conformal to PSC-metrics). Let $\left(M^{n}, g, \mathrm{e}^{-f} \mathrm{dVol}_{g}\right)$ be a closed smooth mmspace with $\mathrm{Sc}_{\alpha, \beta}>0$. If the dimension $n \geq 3, \alpha \in \mathbb{R}$ and $\beta \geq \frac{(n-2)|\alpha|^{2}}{4(n-1)}$, then there is a metric $\tilde{g}$ conformal to $g$ with positive scalar curvature (PSC-metric).

Proof. One only needs to show for all nontrivial $u, \int_{M}-u L_{g} u \mathrm{dVol}_{g}>0$ as in the Yamabe problem [36], where

$$
L_{g}:=\triangle_{g}-\frac{n-2}{4(n-1)} \mathrm{Sc}_{g}
$$


is conformal Laplacian operator. To see this,

$$
\begin{aligned}
\int_{M}-u L_{g} u \mathrm{dVol}_{g}= & \int_{M}\left[\|\nabla g u\|_{g}^{2}+\frac{n-2}{4(n-1)} \mathrm{Sc}_{g} u^{2}\right] \mathrm{dVol}_{g} \\
= & \int_{M}\left[\|\nabla g u\|_{g}^{2}+\frac{n-2}{4(n-1)}\left(\mathrm{Sc}_{\alpha, \beta}-\alpha \triangle_{g} f+\beta\left\|\nabla_{g} f\right\|_{g}^{2}\right) u^{2}\right] \mathrm{dVol}_{g} \\
= & \int_{M}\left[\|\nabla g u\|_{g}^{2}+\frac{n-2}{4(n-1)}\left(\mathrm{Sc}_{\alpha, \beta}+\beta\left\|\nabla_{g} f\right\|_{g}^{2}\right) u^{2}\right. \\
& \left.+\frac{\alpha(n-2)}{2(n-1)}\left\langle\nabla_{g} f, \nabla_{g} u\right\rangle_{g} u\right] \mathrm{dVol}_{g} .
\end{aligned}
$$

Through the inequality

$$
\left\langle\nabla_{g} f, \nabla_{g} u\right\rangle_{g} u \leq c_{2}\left\|\nabla_{g} f\right\|_{g} u \times c_{2}^{-1}\left\|\nabla_{g} u\right\|_{g} \leq \frac{c_{2}^{2}\left\|\nabla_{g} f\right\|_{g}^{2} u^{2}+c_{2}^{-2}\left\|\nabla_{g} u\right\|_{g}^{2}}{2},
$$

one gets

$$
\begin{aligned}
\int_{M}-u L_{g} u \mathrm{dVol}_{g} \geq & \int_{M}\left[\left(1-\frac{|\alpha| c_{2}^{-2}(n-2)}{4(n-1)}\right)\left\|\nabla_{g} u\right\|_{g}^{2}\right. \\
& \left.+\frac{\left(\beta-|\alpha| c_{2}^{-2}\right)(n-2)}{4(n-1)}\|\nabla g f\|_{g}^{2} u^{2}+\frac{n-2}{4(n-1)} \operatorname{Sc}_{\alpha, \beta} u^{2}\right] \mathrm{dVol} g
\end{aligned}
$$

where $c_{2} \neq 0$.

If $|\alpha| c_{2}^{-2} \leq \frac{4(n-1)}{n-2}, \beta \geq c_{2}^{2}|\alpha|$ and $\mathrm{Sc}_{\alpha, \beta}>0$, then

$$
\int_{M}-u L_{g} u \mathrm{dVol}_{g}>0
$$

So the conditions $n>2, \alpha \in \mathbb{R}$ and $\beta \geq \frac{(n-2) \alpha^{2}}{4(n-1)}$ are needed.

Remark 4.8. The proof was borrowed from [1, Proposition 2]. The two propositions above offer a geometric reason why the condition of the vanishing of $\widehat{A}$-genus (without simply connected condition) does not imply that $M^{n}$ can admit a PSC-metric for the closed spin manifold $M^{n}$.

The proposition of conformal to PSC-metrics has following 3 corollaries.

Corollary 4.9 (weighted spherical Lipschitz bounded). Let $\left(M^{n}, g, \mathrm{e}^{-f} \mathrm{dVol}_{g}\right)$ be a closed orientable smooth mm-space with $\mathrm{Sc}_{\alpha, \beta} \geq \kappa>0,3 \leq n \leq 8, \alpha \in \mathbb{R}$ and $\beta \geq \frac{(n-2)|\alpha|^{2}}{4(n-1)}$, then the Lipschitz constant of the continuous map $\phi$ from $\left(M^{n}, g, \mathrm{e}^{-f} \mathrm{dVol}_{g}\right)$ to the sphere $S^{n}$ with standard metric of non-zero degrees has uniformly non-zero lower bounded.

Proof. There is a metric $\tilde{g}$ conformal to $g$ with scalar curvature $\geq n(n-1)$ by the proposition of conformal PSC-metrics. For the continuous map $\phi$ from $\left(M^{n}, \tilde{g}\right)$ to $S^{n}$ of non-zero degrees, the Lipschitz constant of $\phi$ is greater than a constant that depends only on the dimensions $n$ by Gromov's spherical Lipschitz bounded theorem [19, Section 3]. Since the conformal function has the positive upper bound by the compactness of the manifold, then the Lipschitz constant has uniformly non-zero lower bounded.

Corollary 4.10. For the closed smooth mm-space $\left(M^{n}, g, \mathrm{e}^{-f} \mathrm{dVol}_{g}\right)(n \geq 3)$ with $\mathrm{Sc}^{\mathrm{vol}_{n}}\left(M^{n}\right)$ $>0$, there is a metric $\hat{g}$ conformal to $g$ with PSC-metric. In particular, the $\widehat{A}$-genus and Rosenberg index vanish with additional spin condition.

For the closed orientable smooth mm-space $\left(M^{n}, g, \mathrm{e}^{-f} \mathrm{dVol}_{g}\right)(3 \leq n \leq 8)$ with $\operatorname{Sc}^{\operatorname{vol}_{n}}\left(M^{n}\right) \geq$ $\kappa>0$, then the Lipschitz constant of the continuous map $\phi$ from $\left(M^{n}, g, \mathrm{e}^{-f} \mathrm{dVol}_{g}\right)$ to the sphere $S^{n}$ with standard metric of non-zero degrees has uniformly non-zero lower bounded. 
Proof. The volume of the small disk of $\left(M^{n}, g, \mathrm{e}^{-f} \mathrm{dVol}_{g}\right)$ was computed in [33, Theorem 8],

$$
\mu\left(B_{r}(x)\right)=\operatorname{vol}_{E}\left(B_{r}\left(\mathbf{R}^{n}\right)\right)\left[1-\frac{\mathrm{Sc}_{g}+3 \triangle_{g} f-3\left\|\nabla_{g} f\right\|_{g}^{2}}{6(n+2)} r^{2}+O\left(r^{4}\right)\right]
$$

as $r \rightarrow 0$. Since $\operatorname{Sc}^{\operatorname{vol}_{n}}\left(M^{n}\right)>0$, i.e., $\mu\left(B_{r}(x)\right)<\operatorname{vol}_{E}\left(B_{r}\right)$ as $r \rightarrow 0$, then

$$
\mathrm{Sc}_{g}+3 \triangle_{g} f-3\|\nabla g f\|_{g}^{2}>0 .
$$

Therefore, the propositions of vanishing harmonic spinors and of conformal PSC-metrics and Corollary 4.4 imply it.

Remark 4.11. Since any weighted Riemannian manifold (with non-trivial Borel measure) is infinitesimally Hilbertian (see [28]), Corollary 4.10 also works for $\left(M^{n}, g, \mathrm{e}^{-f} \mathrm{dVol}_{g}\right.$ ) with $\operatorname{RSC}(\kappa, n)$ condition.

Enlargeability as an obstruction to the existence of a PSC-metric on a closed manifold was introduced by Gromov-Lawson. We call a manifold enlargeable as Gromov-Lawson's definition in [21, Definition 5.5]

Corollary 4.12. Assume $M^{n}(n \geq 3)$ is a closed spin smooth enlargeable manifold, then $\mathcal{R}_{f}^{+}\left(M^{n}\right)$ is an empty set for $\alpha \in \mathbb{R}$ and $\beta \geq \frac{(n-2)|\alpha|^{2}}{4(n-1)}$.

In particular, $\left(\mathbb{T}^{n}, g, \mathrm{e}^{-f} \mathrm{dVol}_{g}\right)$ does not satisfy $\mathrm{Sc}^{\mathrm{vol}_{n}}\left(\mathbb{T}^{n}\right)>0$ for any $C^{2}$-smooth Riemannian metrics $g$ and $C^{2}$-smooth functions $f$ on the torus $\mathbb{T}^{n}$.

Proof. Since a closed enlargeable manifold cannot carry a PSC-metric [21, Theorem 5.8], Proposition 4.7 implies $\mathcal{R}_{f}^{+}\left(M^{n}\right)=\varnothing$ for $\alpha \in \mathbb{R}$ and $\beta \geq \frac{(n-2)|\alpha|^{2}}{4(n-1)}$.

$\mathbb{T}^{n}$ is an important example of enlargeable manifolds and then Corollary 4.10 implies that $\left(\mathbb{T}^{n}, g, \mathrm{e}^{-f} \mathrm{dVol}_{g}\right)$ does not satisfy $\mathrm{Sc}^{\mathrm{vol}_{n}}\left(\mathbb{T}^{n}\right)>0$ for $n \geq 3$. For dimension 2 , the conditions of $\mathrm{Sc}_{\alpha, \beta}>0$ and $\beta \geq 0$ imply that the oriented surface is 2 -sphere.

\section{$4.3 f$-minimal hypersurface and $\mathrm{Sc}_{\alpha, \beta}>0$}

In addition to using the Dirac operator method, Schoen-Yau's minimal hypersurface method [37] is another main idea. For an immersed orientable hypersurface $N^{n-1} \subset M^{n}$, the weighted mean curvature vector $H_{f}$ of $N^{n-1}$ is defined by Gromov in [16, Section 9.4.E],

$$
H_{f}=H+\left(\nabla_{g} f\right)^{\perp},
$$

where $H$ is the mean curvature vector field of the immersion, $(\cdot)^{\perp}$ is the projection on the normal bundle of $N^{n-1}$. The first and second variational formulae for the weighted volume functional of $N^{n-1}$ were derived in Bayle's thesis (also see [34]). We take the detailed presentation of such derivation for [8]. The $\left(N^{n-1}, \bar{g}\right)$ with the induced metric is called $f$-minimal hypersurface if the weighted mean curvature vector $H_{f}$ vanishes identically.

In fact, the definition of $f$-minimal hypersurface can also be derived from the first variational formula. Furthermore, an $f$-minimal hypersurface is a minimal hypersurface of $\left(M^{n}, \tilde{g}\right)$, where $\tilde{g}$ is the conformal metric of $g, \tilde{g}=\mathrm{e}^{-\frac{2 f}{n-1}} g$.

The connection between the geometry of the ambient smooth mm-space and the $f$-minimal hypersurfaces occurs via the second variation of the weighted volume functional. For a hypersurface $\left(N^{n-1}, \bar{g}\right)$, the $L_{f}$ operator is defined by

$$
L_{f}:=\triangle_{f}+|A|^{2}+\operatorname{Ricc}_{f}^{M}(\nu, \nu),
$$


where $\nu$ is the unit normal vector, $|A|^{2}$ denotes the square of the norm of the second fundamental form $A$ of $N^{n-1}$ and

$$
\triangle_{f}:=\triangle_{\bar{g}}-\left\langle\nabla_{\bar{g}} f, \nabla_{\bar{g}} \cdot\right\rangle
$$

is the weighted Laplacian. Through the second variational formula, a two-sided $f$-minimal hypersurface $N^{n-1}$ is stable (called $L_{f}$-stable) if for any compactly supported smooth function $u \in C_{c}^{\infty}\left(N^{n-1}\right)$, it holds that

$$
-\int_{N} u L_{f} u \mathrm{e}^{-f} \mathrm{dVol}_{\bar{g}} \geq 0 .
$$

Proposition 4.13. Let $\left(M^{n}, g, \mathrm{e}^{-f} \mathrm{dVol}_{g}\right)$ be a closed orientable smooth mm-space with $\mathrm{Sc}_{\alpha, \beta}>0$ and $\left(N^{n-1}, \bar{g}\right)$ be the compact $L_{f}$-stable minimal hypersurface of $\left(M^{n}, g, \mathrm{e}^{-f} \mathrm{dVol}_{g}\right)$. If the dimension $n \geq 3, \alpha=2$, and $\beta \geq \frac{n-2}{n-1}$, then there exists a PSC-metric conformal to $\bar{g}$ on $N^{n-1}$.

Proof. The $f$-minimal hypersurface $\left(N^{n-1}, \bar{g}\right)$ is $L_{f}$-stable if and only if $\left(N^{n-1}, \overline{\tilde{g}}\right)$ is stable as a minimal hypersurface on $\left(M^{n}, \tilde{g}\right)$, where $\tilde{g}:=\mathrm{e}^{-\frac{2 f}{n-1}} g$ and $\overline{\tilde{g}}$ is the induced metric of $\tilde{g}$ (see [8, Appendix]). On the other hand, the scalar curvature of $\left(M^{n}, \tilde{g}\right)$ is

$$
\mathrm{Sc}_{\tilde{g}}=\mathrm{e}^{\frac{f}{n-1}}\left(\mathrm{Sc}_{g}+2 \triangle_{g} f-\frac{n-2}{n-1}\|\nabla g f\|_{g}^{2}\right) .
$$

Thus, $\mathrm{Sc}_{\alpha, \beta}>0$ with $n \geq 3, \alpha=2$, and $\beta \geq \frac{n-2}{n-1}$ imply $\mathrm{Sc}_{\tilde{g}}>0$. Then the standard SchoenYau's argument can be applied to show that $\overline{\tilde{g}}$ conformal to a PSC-metric on $N^{n-1}$.

Remark 4.14. The minimal hypersurface method poses a stricter condition to the valid range of $\alpha, \beta$ than that of the Dirac operator method.

Since the oriented closed manifolds with a PSC-metric in 2 and 3 dimensions are classified by Gauess-Bonnet theorem and Perelman-Thurston geometrization theorem, then Proposition 4.13 can give the following elementary applications:

Corollary 4.15. Let $\left(M^{n}, g, \mathrm{e}^{-f} \mathrm{dVol}_{g}\right)$ be a closed orientable smooth mm-space with $\mathrm{Sc}_{\alpha, \beta}>0$.

1. If $n=3, \alpha=2$, and $\beta \geq \frac{1}{2}$, then there is no closed immersed $L_{f}$-stable minimal 2-dimensional surface with positive genus.

2. If $n=4, \alpha=2$, and $\beta \geq \frac{2}{3}$, then the closed immersed $L_{f}$-stable minimal 3 -dimensional submanifold must be spherical 3-manifolds, $S^{2} \times S^{1}$ or the connected sum of spherical 3-manifolds and copies of $S^{2} \times S^{1}$.

Remark 4.16 (historical remark). The prototype of Corollary 4.15(1) is the Schoen-Yau's classic result, which said that the oriented closed 3-manifold with a PSC-metric has no compact immersed stable minimal surface of positive genus [37]. The Schoen-Yau result had been generalized to Perelman's P-scalar curvature $>0$ by Fan [10]. Note that one can also consider the noncompact immersed $L_{f}$-stable minimal 2-dimensional surface under the condition of Corollary 4.15(1) since an oriented complete stable minimal surface in a complete oriented 3 -manifold with a PSC-metric is conformally equivalent to the complex plane $\mathbb{C}$ showed by Fischer-Colbrie-Schoen [11].

The smooth mm-space with $\mathrm{Sc}_{\alpha, \beta}>0$ under suit ranges of $\alpha$ and $\beta$ implies the manifold admits PSC-metrics, but the manifold (itself) that can admit PSC-metrics does not necessarily imply there exists $\mathrm{Sc}_{\alpha, \beta}>0$. 
Question 4.17. Does the smooth mm-space with $\mathrm{Sc}_{\alpha, \beta}>0$ under suitable ranges of $\alpha$ and $\beta$ give more topological restriction on the manifold than the PSC-metric on the manifold?

Question 4.18. Let $M$ be a closed smooth manifold, $f$ be a smooth function on $M$ and $h$ be a smooth function that is negative for some point $p$ on $M$. What is the range of $\alpha$ and $\beta$ such that there exists a smooth Riemannian metric $g$ on $M$ satisfying

$$
\mathrm{Sc}_{g}+\alpha \triangle_{g} f-\beta\left\|\nabla_{g} f\right\|_{g}^{2}=h,
$$

i.e., $\mathrm{Sc}_{\alpha, \beta}(g)=h$ ?

Let $\left(M, g_{i}\right)$ be smooth Riemannian manifolds and $\left\{g_{i}\right\}_{i \in \mathbf{N}} C^{0}$-converges to $g$, then $\left\{g_{i}\right\}_{i \in \mathbf{N}}$ also smGH-converges to $g$. Gromov showed that the scalar curvature $\geq \kappa$ is stable under $C^{0}$ convergence in [17, Section 1.8].

Question 4.19. Assume smooth mm-spaces $\left(M^{n}, g_{i}, \mathrm{e}^{-f} \mathrm{dVol}_{g_{i}}\right)$ all satisfy $\mathrm{Sc}^{\operatorname{vol}_{n}}\left(M^{n}\right) \geq 0$ such that $\left\{g_{i}\right\}_{i \in \mathbf{N}} C^{2}$-converges to the smooth Riemannian metric $g$ on $M^{n}$, then does $\left(M^{n}, g\right.$, $\left.\mathrm{e}^{-f} \mathrm{dVol}_{g}\right)$ also satisfy $\mathrm{Sc}^{\operatorname{vol}_{n}}\left(M^{n}\right) \geq 0$ ?

Question 4.20. Let mm-spaces $\left(M^{n}, g, \mathrm{e}^{-f} \mathrm{dVol}_{g}\right)$ with $\mathrm{Sc}^{\mathrm{vol}_{n}}\left(M^{n}\right) \geq \kappa>0$, where $M^{n}$ is a closed smooth manifold, $g$ and $f$ are $C^{0}$-smooth, then does there exist a PSC-metric on $M^{n}$ ?

Since the role of $\mathrm{Sc}_{\alpha, \beta}>0$ on the smooth mm-space is similar to the role of $\mathrm{Sc}>0$ on the manifold, one can try to extend the knowledge about $\mathrm{Sc}>0$ to $\mathrm{Sc}_{\alpha, \beta}>0$.

\subsection{Weighted rigidity}

Gromov's conjecture that said if a smooth Riemannian metric $g$ satisfies $g \geq g_{\text {st }}$ and $\operatorname{Sc}(g) \geq$ $\operatorname{Sc}\left(g_{\mathrm{st}}\right)=n(n-1)$ on $S^{n}$ then $g=g_{\mathrm{st}}$, was proved by Llarull [27] and called Llarull rigidity theorem. A map $h:\left(M^{n}, g_{M}\right) \rightarrow\left(N^{n}, g_{N}\right)$ is said to be $\epsilon$-contracting if $\left\|h_{*} v\right\|_{g_{N}} \leq \epsilon\|v\|_{g_{N}}$ for all tangent vectors $v$ on $M^{n}$

Proposition 4.21 (weighted rigidity). Assume the smooth mm-space $\left(M^{n}, g, \mathrm{e}^{-f} \mathrm{dVol}_{g}\right)$ is closed and spin and there exists a smooth 1-contracting map $h:\left(M^{n}, g\right) \rightarrow\left(S^{n}, g_{\mathrm{st}}\right)$ of nonzero degree. If $\alpha \in \mathbb{R}, \beta \geq \frac{|\alpha|^{2}}{4}$ and $\mathrm{Sc}_{\alpha, \beta} \geq n(n-1)$, then $h$ is an isometry between the metrics $g$ and $g_{\mathrm{st}}$. Furthermore, if $\alpha>0$, then $f$ is a constant function.

Proof. One just need to insert the tricks in the proof of Proposition 4.3 to the proof in [27, Theorem 4.1]. Following the setup of Llarull, we only prove the even-dimensional $(2 n)$ case without loss of generality.

First, we will show that $h$ is an isometry. Fix $p \in M^{2 n}$. Let $\left\{e_{1}, \ldots, e_{2 n}\right\}$ be a $g$-orthonormal tangent fame near $p$ such that $\left(\nabla_{g} e_{k}\right)_{p}=0$ for each $k$. Let $\left\{\epsilon_{1}, \ldots, \epsilon_{2 n}\right\}$ be a $g_{\text {st }}$-orthonormal tangent frame near $h(p) \in S^{2 n}$ such that $\left(\nabla g_{\mathrm{st}} \epsilon_{k}\right)_{h(p)}=0$ for each $k$. Moreover, the bases $\left\{e_{1}, \ldots, e_{2 n}\right\}$ and $\left\{\epsilon_{1}, \ldots, \epsilon_{2 n}\right\}$ can be chosen so that $\epsilon_{j}=\lambda_{j} h_{*} e_{j}$ for appropriate $\left\{\lambda_{j}\right\}_{j=1}^{2 n}$. This is possible since $h_{*}$ is symmetric. Since $h$ is 1-contracting map, $\lambda_{k} \geq 1$ for each $k$.

Then one constructs the twisted vector bundles $S \otimes E$ over $M^{2 n}$ as Llarull did. Let $R^{E}$ be the curvature tensor of $E$ and $\psi$ be a twisted spinor, then one gets

$$
\left\langle R^{E} \psi, \psi\right\rangle_{g} \geq-\frac{1}{4} \sum_{i \neq j} \frac{1}{\lambda_{i} \lambda_{j}}\|\psi\|_{g}
$$


For the twisted Dirac operator $\mathbb{D}_{E}$, one has $\mathbb{D}_{E}^{2}=\nabla^{*} \nabla+\frac{1}{4} \mathrm{Sc}_{g}+R^{E}$ and

$$
\begin{aligned}
\int_{M}\left\langle\mathbb{D}_{E}^{2} \psi, \psi\right\rangle_{g} \mathrm{dVol}_{g}= & \int_{M}\left[\|\nabla g \psi\|_{g}^{2}+\frac{1}{4}\left(\mathrm{Sc}_{\alpha, \beta}-\alpha \triangle_{g} f+\beta\left\|\nabla_{g} f\right\|_{g}^{2}\right)\|\psi\|_{g}^{2}\right. \\
& \left.+\left\langle R^{E} \psi, \psi\right\rangle_{g}\right] \mathrm{dVol}_{g} \\
= & \int_{M}\left[\left\|\nabla_{g} \psi\right\|_{g}^{2}+\left(\frac{1}{4} \mathrm{Sc}_{\alpha, \beta}+\frac{\beta}{4}\left\|\nabla_{g} f\right\|_{g}^{2}\right)\|\psi\|_{g}^{2}\right. \\
& \left.+\frac{\alpha}{4}\left\langle\nabla_{g} f, \nabla_{g}\|\psi\|_{g}^{2}\right\rangle_{g}+\left\langle R^{E} \psi, \psi\right\rangle_{g}\right] \mathrm{dVol}_{g} .
\end{aligned}
$$

Because $\lambda_{k} \geq 1$ for each $k$, one gets

$$
\left\langle R^{E} \psi, \psi\right\rangle_{g} \geq \frac{-2 n(2 n-1)}{4}\|\psi\|_{g}
$$

and then

$$
\begin{aligned}
\frac{|\alpha|}{4}\left|\left\langle\nabla_{g} f, \nabla_{g}\|\psi\|_{g}^{2}\right\rangle_{g}\right| & \leq \frac{|\alpha|}{4}\left(\left\|\nabla_{g} f\right\|_{g}\|\psi\|_{g} \times 2\left\|\nabla_{g} \psi\right\|_{g}\right) \\
& =\frac{|\alpha|}{2}\left(c_{1}\left\|\nabla_{g} f\right\|_{g}\|\psi\|_{g} \times c_{1}^{-1}\left\|\nabla_{g} \psi\right\|_{g}\right) \\
& \leq \frac{|\alpha|}{4}\left(c_{1}^{2}\left\|\nabla_{g} f\right\|_{g}^{2}\|\psi\|_{g}^{2}+c_{1}^{-2}\left\|\nabla_{g} \psi\right\|_{g}^{2}\right),
\end{aligned}
$$

where $c_{1} \neq 0$. Therefore,

$$
\begin{aligned}
\int_{M}\left\langle\mathbb{D}_{E}^{2} \psi, \psi\right\rangle_{g} \mathrm{dVol}_{g} \geq & \int_{M}\left[\left(1-\frac{c_{1}^{-2}|\alpha|}{4}\right)\|\nabla g \psi\|_{g}^{2}+\frac{\beta-c_{1}^{2}|\alpha|}{4}\|\nabla g f\|_{g}^{2}\|\psi\|_{g}^{2}\right. \\
& \left.+\frac{1}{4}\left(\operatorname{Sc}_{\alpha, \beta}-2 n(2 n-1)\right)\|\psi\|_{g}^{2}\right] \mathrm{dVol}_{g} .
\end{aligned}
$$

Furthermore, since $\alpha \in \mathbb{R}, \beta \geq \frac{|\alpha|^{2}}{4}$ and $\mathrm{Sc}_{\alpha, \beta} \geq 2 n(2 n-1)$, one can choose $c_{1}$ such that $c_{1}^{-2}|\alpha| \leq 4$, then $\beta-c_{1}^{2}|\alpha| \geq 0$. Thus,

$$
\int_{M}\left\langle\mathbb{D}_{E}^{2} \psi, \psi\right\rangle_{g} \mathrm{dVol}_{g} \geq \int_{M} \frac{1}{4}\left[\mathrm{Sc}_{\alpha, \beta}-2 n(2 n-1)\right]\|\psi\|_{g}^{2} \mathrm{dVol}_{g} \geq 0 .
$$

The fact $\operatorname{Index}\left(\mathbb{D}_{E^{+}}\right) \neq 0$ implies $\operatorname{ker}\left(\mathbb{D}_{E}\right) \neq 0$ and then $\operatorname{Sc}_{\alpha, \beta}=2 n(2 n-1)$. Then using the inequality $\left\langle R^{E} \psi, \psi\right\rangle_{g} \geq-\frac{1}{4} \sum_{i \neq j} \frac{1}{\lambda_{i} \lambda_{j}}\|\psi\|_{g}$, one gets

$$
\int_{M}\left\langle\mathbb{D}_{E}^{2} \psi, \psi\right\rangle_{g} \mathrm{dVol}_{g} \geq \int_{M} \frac{1}{4}\left[\sum_{i \neq j}\left(1-\frac{1}{\lambda_{i} \lambda_{j}}\right)\right]\|\psi\|_{g}^{2} \mathrm{dVol}_{g} \geq 0 .
$$

Choosing $\psi \neq 0$ such that $\mathbb{D}_{E} \psi=0$, one has

$$
0 \leq 1-\frac{1}{\lambda_{i} \lambda_{j}} \leq 0
$$

for $i \neq j$. Thus, $\lambda_{k}=0$ for all $1 \leq k \leq 2 n$ and $h$ is an isometry.

Second, we will show that $f$ is a constant function. Since $\mathrm{Sc}_{\alpha, \beta}=2 n(2 n-1), \mathrm{Sc}_{g}=2 n(2 n-1)$, $\alpha>0$ and $\beta \geq \frac{|\alpha|^{2}}{4}$, then $\triangle_{g} f \geq 0$. One has

$$
\int_{M} \triangle_{g} f \mathrm{dVol}_{g}=0
$$

for a closed manifold $M^{n}$, so one gets $\triangle_{g} f=0$. That implies $\nabla_{g} f=0$ so that $f$ is a constant function on $M^{n}$. 
Corollary 4.22. Let the closed and spin smooth mm-space $\left(M^{n}, g, \mathrm{e}^{-f} \mathrm{dVol}_{g}\right)$ with $\mathrm{Sc}^{\operatorname{vol}_{n}}\left(M^{n}\right)$ $\geq n(n-1)$ and there exists a smooth 1-contracting map $h:\left(M^{n}, g\right) \rightarrow\left(S^{n}, g_{\mathrm{st}}\right)$ of non-zero degree, then $h$ is an isometry between the metrics $g$ and $g_{\mathrm{st}}$.

Proof. Combining the weighted rigidity theorem and the proof of Corollary 4.10 can imply it.

As Llarull rigidity theorem (and the weighted rigidity theorem) still holds if the condition that $h$ is 1 -contracting is replaced by the condition that $h$ is area-contracting, Gromov called such metrics area-extremal metrics, asked which manifolds possess area-extremal metrics, and conjectured that Riemannian symmetric spaces should have area-extremal metrics [15], [18, Section 17] and [20, Section 4.2]. Goette-Semmelmann showed that several classes of symmetric spaces with non-constant curvatures are area-extremal [13].

Question 4.23. Can Goette-Semmelmann's results [13] be generalized to the smooth mm-space with $\mathrm{Sc}_{\alpha, \beta}>0$ under other suitable conditions?

Since Corollary 4.15(1) showed that the closed orientable immersed $L_{f}$-stable minimal 2dimensional surface in the closed orientable smooth mm-space $\left(M^{n}, g, \mathrm{e}^{-f} \mathrm{dVol}_{g}\right)$ with $\mathrm{Sc}_{2, \beta}>0$ $\left(\beta \geq \frac{1}{2}\right)$ is 2 -sphere, then one can consider rigidity of area-minimizing 2-sphere in 3-dimensional smooth mm-space. Bray's volume comparison theorem [4, Chapter 3, Theorem 18] is another rigidity theorem that needs the conditions of Ricci curvature and scalar curvature bounded below. There are other rigidity phenomena involving scalar curvature, see [5].

Question 4.24. Can Bray's volume comparison theorem be extended to the smooth mm-space?

Question 4.25. What is the correct Einstein field equation on the smooth mm-space?

If one replaces the Ricci and scalar curvature on the left hand side of Einstein field question by $\operatorname{Ricc}_{f}^{M}$ and $\mathrm{Sc}_{\alpha, \beta}$ for the smooth mm-space $\left(M^{n}, g, \mathrm{e}^{-f} \mathrm{dVol}_{g}\right)$, then what is the stress-energy tensor on the right hand side in this case?

\section{Acknowledgements}

I am grateful to Thomas Schick for his help, the referees for their useful comments, and the funding from China Scholarship Council.

\section{References}

[1] Abedin F., Corvino J., On the P-scalar curvature, J. Geom. Anal. 27 (2017), 1589-1623.

[2] Ambrosio L., Gigli N., Savaré G., Metric measure spaces with Riemannian Ricci curvature bounded from below, Duke Math. J. 163 (2014), 1405-1490, arXiv:1109.0222.

[3] Athreya S., Löhr W., Winter A., The gap between Gromov-vague and Gromov-Hausdorff-vague topology, Stochastic Process. Appl. 126 (2016), 2527-2553, arXiv:1407.6309.

[4] Bray H.L., The Penrose inequality in general relativity and volume comparison theorems involving scalar curvature, Ph.D. Thesis, Stanford University, 1997, arXiv:0902.3241.

[5] Brendle S., Rigidity phenomena involving scalar curvature, in Surveys in Differential Geometry, Surv. Differ. Geom., Vol. 17, Int. Press, Boston, MA, 2012, 179-202, arXiv:1008.3097.

[6] Case J.S., Conformal invariants measuring the best constants for Gagliardo-Nirenberg-Sobolev inequalities, Calc. Var. Partial Differential Equations 48 (2013), 507-526, arXiv:1112.3977.

[7] Chang S.-Y.A., Gursky M.J., Yang P., Conformal invariants associated to a measure, Proc. Natl. Acad. Sci. USA 103 (2006), 2535-2540.

[8] Cheng X., Mejia T., Zhou D., Stability and compactness for complete $f$-minimal surfaces, Trans. Amer. Math. Soc. 367 (2015), 4041-4059, arXiv:1210.8076. 
[9] Crowley D., Schick T., The Gromoll filtration, $K O$-characteristic classes and metrics of positive scalar curvature, Geom. Topol. 17 (2013), 1773-1789, arXiv:1204.6474.

[10] Fan E.M., Topology of three-manifolds with positive P-scalar curvature, Proc. Amer. Math. Soc. 136 (2008), $3255-3261$.

[11] Fischer-Colbrie D., Schoen R., The structure of complete stable minimal surfaces in 3-manifolds of nonnegative scalar curvature, Comm. Pure Appl. Math. 33 (1980), 199-211.

[12] Gigli N., On the differential structure of metric measure spaces and applications, Mem. Amer. Math. Soc. 236 (2015), vi+91 pages, arXiv:1205.6622.

[13] Goette S., Semmelmann U., Scalar curvature estimates for compact symmetric spaces, Differential Geom. Appl. 16 (2002), 65-78, arXiv:math.DG/0010199.

[14] Gray A., Vanhecke L., Riemannian geometry as determined by the volumes of small geodesic balls, Acta Math. 142 (1979), 157-198.

[15] Gromov M., Positive curvature, macroscopic dimension, spectral gaps and higher signatures, in Functional Analysis on the Eve of the 21st Century, Vol. II (New Brunswick, NJ, 1993), Progr. Math., Vol. 132, Birkhäuser Boston, Boston, MA, 1996, 1-213.

[16] Gromov M., Isoperimetry of waists and concentration of maps, Geom. Funct. Anal. 13 (2003), $178-215$.

[17] Gromov M., Dirac and Plateau billiards in domains with corners, Cent. Eur. J. Math. 12 (2014), 1109-1156, arXiv:1811.04318.

[18] Gromov M., 101 questions, problems and conjectures around scalar curvature, 2017, available at https: //www. ihes.fr/ gromov/wp-content/uploads/2018/08/101-problems0ct1-2017.pdf.

[19] Gromov M., Metric inequalities with scalar curvature, Geom. Funct. Anal. 28 (2018), 645-726, arXiv:1710.04655.

[20] Gromov M., Four lectures on scalar curvature, arXiv:1908.10612.

[21] Gromov M., Lawson Jr. H.B., Positive scalar curvature and the Dirac operator on complete Riemannian manifolds, Inst. Hautes Études Sci. Publ. Math. 58 (1983), 83-196.

[22] Hanke B., Pape D., Schick T., Codimension two index obstructions to positive scalar curvature, Ann. Inst. Fourier (Grenoble) 65 (2015), 2681-2710, arXiv:1402.4094.

[23] Hitchin N., Harmonic spinors, Adv. Math. 14 (1974), 1-55.

[24] Kazdan J.L., Deformation to positive scalar curvature on complete manifolds, Math. Ann. 261 (1982), $227-234$.

[25] Kazdan J.L., Warner F.W., Scalar curvature and conformal deformation of Riemannian structure, J. Differential Geometry 10 (1975), 113-134.

[26] Lasserre J.B., On the setwise convergence of sequences of measures, J. Appl. Math. Stochastic Anal. 10 (1997), 131-136.

[27] Llarull M., Sharp estimates and the Dirac operator, Math. Ann. 310 (1998), 55-71.

[28] Lučić D., Pasqualetto E., Infinitesimal Hilbertianity of weighted Riemannian manifolds, Canad. Math. Bull. 63 (2020), 118-140, arXiv:1809.05919.

[29] Ohta S., On the measure contraction property of metric measure spaces, Comment. Math. Helv. 82 (2007), 805-828.

[30] Ohta S., Finsler interpolation inequalities, Calc. Var. Partial Differential Equations 36 (2009), 211-249.

[31] Perelman G., The entropy formula for the Ricci flow and its geometric applications, arXiv:math.DG/0211159.

[32] Petrunin A., Alexandrov meets Lott-Villani-Sturm, Münster J. Math. 4 (2011), 53-64, arXiv:1003.5948.

[33] Qian Z., Estimates for weighted volumes and applications, Quart. J. Math. Oxford Ser. (2) 48 (1997), 235-242.

[34] Rosales C., Cañete A., Bayle V., Morgan F., On the isoperimetric problem in Euclidean space with density, Calc. Var. Partial Differential Equations 31 (2008), 27-46, arXiv:math.DG/0602135.

[35] Rosenberg J., $C^{*}$-algebras, positive scalar curvature, and the Novikov conjecture, Inst. Hautes Études Sci. Publ. Math. 58 (1983), 197-212.

[36] Schoen R., Conformal deformation of a Riemannian metric to constant scalar curvature, J. Differential Geom. 20 (1984), 479-495. 
[37] Schoen R., Yau S.T., Existence of incompressible minimal surfaces and the topology of three-dimensional manifolds with nonnegative scalar curvature, Ann. of Math. 110 (1979), 127-142.

[38] Sturm K.-T., On the geometry of metric measure spaces. II, Acta Math. 196 (2006), 133-177.

[39] Villani C., Optimal transport: old and new, Grundlehren der Mathematischen Wissenschaften, Vol. 338, Springer-Verlag, Berlin, 2009.

[40] Wylie W., Sectional curvature for Riemannian manifolds with density, Geom. Dedicata 178 (2015), 151-169, arXiv:1311.0267.

[41] Young L.S., Dimension, entropy and Lyapunov exponents, Ergodic Theory Dynam. Systems 2 (1982), 109124.

[42] Zeidler R., An index obstruction to positive scalar curvature on fiber bundles over aspherical manifolds, Algebr. Geom. Topol. 17 (2017), 3081-3094, arXiv:1512.06781.

[43] Zhang H.-C., Zhu X.-P., Ricci curvature on Alexandrov spaces and rigidity theorems, Comm. Anal. Geom. 18 (2010), 503-553, arXiv:0912.3190. 